\title{
MORSE INEQUALITIES FOR FOURIER COMPONENTS OF KOHN-ROSSI COHOMOLOGY OF CR COVERING MANIFOLDS WITH $S^{1}$-ACTION
}

\author{
RUNG-TZUNG HUANG AND GUOKUAN SHAO
}

\begin{abstract}
Let $X$ be a compact connected CR manifold of dimension $2 n+1, n \geq 1$. Let $\widetilde{X}$ be a paracompact CR manifold with a transversal CR $S^{1}$-action, such that there is a discrete group $\Gamma$ acting freely on $\widetilde{X}$ having $X=\widetilde{X} / \Gamma$. Based on an asymptotic formula for the Fourier components of the heat kernel with respect to the $S^{1}$-action, we establish the Morse inequalities for Fourier components of reduced $L^{2}$-Kohn-Rossi cohomology with values in a rigid CR vector bundle over $\widetilde{X}$. As a corollary, we obtain the Morse inequalities for Fourier components of Kohn-Rossi cohomology on $X$ which were obtained by Hsiao-Li [16] by using Szegö kernel method.
\end{abstract}

\section{Introduction AND STATEMENT OF the RESUlts}

Gromov-Henkin-Shubin [11, Theorem 0.2] considered covering manifolds that are strongly pseudoconvex of complex manifolds and analyzed the holomorphic $L^{2}$-functions on the coverings. Todor-Chiose-Marinescu [24] generalized in a similar manner the Morse inequalities of Siu-Demailly [22, 7] on coverings of complex manifolds, they also considered coverings of weakly pseudoconvex domains in [20]. The study of problems on CR manifolds with $S^{1}$-action becomes active recently, see [5, 12, 13, 15, 16, 18] and the references therein. In particular, Hsiao-Li [16] established the Morse inequalities for Fourier components of Kohn-Rossi cohomology on $X$ by using the Szegö kernel method. In [17] general Morse inequalities for CR bundles are proved, generalizing [9]. Inspired by the results of [11, 16, 24, 22, 7], we establish Morse inequalities for Fourier components of reduced $L^{2}$-Kohn-Rossi cohomology with values in a rigid CR vector bundle on a covering manifold over a compact connected CR manifold with $S^{1}$-action. This generalizes the results of [16] to CR covering manifolds with $S^{1}$-action. We present a proof by the heat kernel method, which is inspired by Bismut's proof [3, 19] of the holomorphic Morse inequalities. The crucial estimate for Fourier components of the heat kernel of Kohn Laplacians was given in [13].

Now we formulate the main results. We refer to other sections for notations and definitions (see Definition 2.1, 2.2, 2.3, 2.5 and (3.1), (3.25) ) used here. Let $X$ be a compact connected CR manifold of dimension $2 n+1, n \geq 1$ with a transversal CR $S^{1}$-action $e^{i \theta}$ on $X$. For $x \in X$, we say that the period of $x$ is $\frac{2 \pi}{\ell}, \ell \in \mathbb{N}$, if $e^{i \theta} \circ x \neq x$, for every $0<\theta<\frac{2 \pi}{\ell}$, and $e^{i \frac{2 \pi}{\ell}} \circ x=x$. For each $\ell \in \mathbb{N}$, put

$$
X_{\ell}=\left\{x \in X ; \text { the period of } x \text { is } \frac{2 \pi}{\ell}\right\}
$$

and let

$$
p=\min \left\{\ell \in \mathbb{N} ; X_{\ell} \neq \emptyset\right\}
$$

2010 Mathematics Subject Classification. 58J35, 32V20.

Key words and phrases. Kohn-Rossi cohomology, heat kernel, CR manifold.

The first author was supported by Taiwan Ministry of Science and Technology project 107-2115-M-008-007MY2. Both authors would like to express their gratitude to Prof. Chin-Yu Hsiao for very helpful comments in this work. 
It is well-known that if $X$ is connected, then $X_{p}$ is an open and dense subset of $X$ (see Duistermaat-Heckman [6, p.265]). Assume $X=X_{p_{1}} \cup X_{p_{2}} \cup \cdots \cup X_{p_{k}}, p=: p_{1}<p_{2}<\cdots<p_{k}$. Set $X_{\text {reg }}:=X_{p}$. We call $x \in X_{\text {reg }}$ a regular point of the $S^{1}$ action. Let $X_{\text {sing }}$ be the complement of $X_{\text {reg }}$.

Let $\widetilde{X}$ be a paracompact CR manifold, such that there is a discrete group $\Gamma$ acting freely on $\widetilde{X}$ having $X=\widetilde{X} / \Gamma$. Let $\pi: \widetilde{X} \rightarrow X$ be the natural projection with the pull-back map $\pi^{*}: T X \rightarrow T \widetilde{X}$. Then $\widetilde{X}$ admits a pull-back CR structure $T^{1,0} \widetilde{X}:=\pi^{*} T^{1,0} X$ and, hence, a CR manifold. We assume that $\widetilde{X}$ admits a transversal CR locally free $S^{1}$ action, denote by $e^{i \theta}$. We further assume that the map

$$
\Gamma \times \widetilde{X} \rightarrow \widetilde{X},(\gamma, \widetilde{x}) \mapsto \gamma \circ \widetilde{x}, \quad \forall \widetilde{x} \in \widetilde{X}, \quad \forall \gamma \in \Gamma .
$$

is $\mathrm{CR}$, see (2.6), and

$$
e^{i \theta} \circ \gamma \circ \widetilde{x}=\gamma \circ e^{i \theta} \circ \widetilde{x}, \quad \forall \widetilde{x} \in \widetilde{X}, \quad \forall \theta \in[0,2 \pi[, \quad \forall \gamma \in \Gamma .
$$

Let $\widetilde{E}:=\pi^{*} E$ be the pull-pack bundle of a rigid CR vector bundle $E$ over $X$. Then $\widetilde{E}$ is a $\Gamma$-invariant rigid $\mathrm{CR}$ vector bundle over $\widetilde{X}$. We denote by $\widetilde{X}_{\mathrm{reg}}$ the set of regular points of the $S^{1}$-action on $\tilde{X}$. Note that since $\Gamma$ acts on $\tilde{X}$ freely so that $\tilde{X} / \Gamma=X$, hence, we have $\widetilde{X}_{\text {reg }} / \Gamma=X_{\text {reg }}=X_{p}$. We denote by $X(q)$ a subset of $X$ such that

$X(q):=\left\{x \in X: \mathcal{L}_{x}\right.$ has exactly $q$ negative eigenvalues and $n-q$ positive eigenvalues $\}$.

We refer to Section 2 for more details. Our main theorem is the following

Theorem 1.1. With the above notations and assumptions, as $m \rightarrow \infty$, for $q=0,1, \cdots, n$, the $m$-th Fourier components of reduced $L^{2}$-Kohn-Rossi cohomology (see (3.25)) satisfy the following strong Morse inequalities

$$
\begin{aligned}
& \sum_{j=0}^{q}(-1)^{q-j} \operatorname{dim}_{\Gamma} \bar{H}_{b,(2), m}^{j}(\widetilde{X}, \widetilde{E}) \\
& \leq \frac{p r m^{n}}{2 \pi^{n+1}} \sum_{j=0}^{q}(-1)^{q-j} \int_{X(j)}\left|\operatorname{det}\left(\mathcal{L}_{x}\right)\right| d v_{X}(x)+o\left(m^{n}\right), \text { for } p \mid m, \\
& \sum_{j=0}^{q}(-1)^{q-j} \operatorname{dim}_{\Gamma} \bar{H}_{b,(2), m}^{j}(\widetilde{X}, \widetilde{E})=o\left(m^{n}\right), \text { for } p \nmid m .
\end{aligned}
$$

where $r$ denotes the rank of $\widetilde{E}, \operatorname{dim}_{\Gamma}$ denotes the Von Neumann dimension (see $\$ 2.3$ in the below, [19, §3.6.1] or [1, §3]) and $\mathcal{L}_{x}$ is the Levi form at $x \in X$. When $p \mid m, q=n$, as $m \rightarrow \infty$, we have the asymptotic Riemann-Roch-Hirzebruch theorem

$$
\sum_{j=0}^{n}(-1)^{j} \operatorname{dim}_{\Gamma} \bar{H}_{b,(2), m}^{j}(\widetilde{X}, \widetilde{E})=\frac{p r m^{n}}{2 \pi^{n+1}} \sum_{j=0}^{n}(-1)^{j} \int_{X(j)}\left|\operatorname{det}\left(\mathcal{L}_{x}\right)\right| d v_{X}(x)+o\left(m^{n}\right) .
$$

In particular, we get the weak Morse inequalities

$$
\operatorname{dim}_{\Gamma} \bar{H}_{b,(2), m}^{q}(\widetilde{X}, \widetilde{E}) \leq \frac{p r m^{n}}{2 \pi^{n+1}} \int_{X(q)}\left|\operatorname{det}\left(\mathcal{L}_{x}\right)\right| d v_{X}(x)+o\left(m^{n}\right) .
$$

By the standard argument in [16] or [14], we deduce easily the following Grauert-Riemenschneider criterion on coverings of CR manifolds.

Corollary 1.2. With the above notations and assumptions in Theorem 1.1, we assume also that $X$ is weakly pseudoconvex and strongly pseudoconvex at a point. Then

$$
\operatorname{dim}_{\Gamma} \bar{H}_{b,(2), m}^{0}(\widetilde{X}, \widetilde{E}) \approx m^{n}, \text { for } p \mid m .
$$


In particular, $\operatorname{dim}_{\Gamma} \bar{H}_{b,(2)}^{0}(\widetilde{X}, \widetilde{E})=\infty$.

When $\Gamma=\{e\}, p=1$ and $\widetilde{E}$ is trivial line bundle, we deduce the following Morse inequalities of Hsiao-Li, see [16, Theorem 2.2 and Theorem 2.5].

Corollary 1.3. With the above notations and assumptions, as $m \rightarrow \infty$, for $q=0,1, \cdots, n$, the m-th Fourier components of Kohn-Rossi cohomology satisfy the following strong Morse inequalities,

$$
\sum_{j=0}^{q}(-1)^{q-j} \operatorname{dim} H_{b, m}^{j}(X) \leq \frac{m^{n}}{2 \pi^{n+1}} \sum_{j=0}^{q}(-1)^{q-j} \int_{X(j)}\left|\operatorname{det}\left(\mathcal{L}_{x}\right)\right| d v_{X}(x)+o\left(m^{n}\right),
$$

where $\mathcal{L}_{x}$ is the Levi form at $x \in X$. In particular, we get the weak Morse inequalities

$$
\operatorname{dim} H_{b, m}^{q}(X) \leq \frac{m^{n}}{2 \pi^{n+1}} \int_{X(q)}\left|\operatorname{det}\left(\mathcal{L}_{x}\right)\right| d v_{X}(x)+o\left(m^{n}\right) .
$$

Let $X$ be a compact CR manifold of dimension $2 n+1, n \geq 1$. A classical theorem due to Boutet de Monvel [4] asserts that $X$ can be globally CR embedded into $\mathbb{C}^{N}$, for some $N \in \mathbb{N}$, when $X$ is strongly pseudoconvex with dimension $n \geq 5$. Epstein [8] proved that if $X$ is strongly pseudoconvex with dimension 3 and a global free transversal CR $S^{1}$-action, then $X$ can be embedded into $\mathbb{C}^{N}$ by positive Fourier components of $\mathrm{CR}$ functions. Corollary 1.3 guarantees the abundance of positive Fourier components of CR functions to do embedding in general cases (e.g. the $S^{1}$-action can be only locally free). In [16], the authors' proofs include localization of analytic objects (eigenfunctions, Szegö kernels), Kohn $L^{2}$ estimates and scaling techniques. A more general version of Corollary 1.3 (with $X$ being weakly pseudoconvex) is proved by Cheng-Hsiao-Tsai in [5, Proposition 1.20 and Corollary 1.21] in a different way. By using the Morse inequalities, (1.7) and (1.8), Hsiao-Li [16, Theorem 2.6] proved that there are abundant CR functions on $X$ when $X$ is weakly pseudoconvex and strongly pseudoconvex at a point. Corollary 1.2 generalizes Theorem 2.6 of [16] to CR covering manifolds.

This paper is organized as follows. In Section 2 we introduce some basic notations, terminology and definitions. In Section 3 we study the asymptotic behavior of heat kernels of Kohn Laplacians. Section 4 is devoted to the heat kernel proof of the main theorem.

\section{Preliminaries}

2.1. Some standard notations. We use the following notations: $\mathbb{N}=\{1,2, \ldots\}, \mathbb{N}_{0}=$ $\mathbb{N} \cup\{0\}, \mathbb{R}$ is the set of real numbers, $\mathbb{R}_{+}:=\{x \in \mathbb{R} ; x>0\}, \overline{\mathbb{R}}_{+}:=\{x \in \mathbb{R} ; x \geq 0\}$. For a multiindex $\alpha=\left(\alpha_{1}, \ldots, \alpha_{n}\right) \in \mathbb{N}_{0}^{n}$ we set $|\alpha|=\alpha_{1}+\cdots+\alpha_{n}$. For $x=\left(x_{1}, \ldots, x_{n}\right)$ we write

$$
x^{\alpha}=x_{1}^{\alpha_{1}} \ldots x_{n}^{\alpha_{n}}, \quad \partial_{x_{j}}=\frac{\partial}{\partial x_{j}}, \quad \partial_{x}^{\alpha}=\partial_{x_{1}}^{\alpha_{1}} \ldots \partial_{x_{n}}^{\alpha_{n}}=\frac{\partial^{|\alpha|}}{\partial x^{\alpha}} .
$$

Let $z=\left(z_{1}, \ldots, z_{n}\right), z_{j}=x_{2 j-1}+i x_{2 j}, j=1, \ldots, n$, be coordinates of $\mathbb{C}^{n}$. We write

$$
\begin{aligned}
& z^{\alpha}=z_{1}^{\alpha_{1}} \ldots z_{n}^{\alpha_{n}}, \quad \bar{z}^{\alpha}=\bar{z}_{1}^{\alpha_{1}} \ldots \bar{z}_{n}^{\alpha_{n}}, \\
& \partial_{z_{j}}=\frac{\partial}{\partial z_{j}}=\frac{1}{2}\left(\frac{\partial}{\partial x_{2 j-1}}-i \frac{\partial}{\partial x_{2 j}}\right), \quad \partial_{\bar{z}_{j}}=\frac{\partial}{\partial \bar{z}_{j}}=\frac{1}{2}\left(\frac{\partial}{\partial x_{2 j-1}}+i \frac{\partial}{\partial x_{2 j}}\right), \\
& \partial_{z}^{\alpha}=\partial_{z_{1}}^{\alpha_{1}} \ldots \partial_{z_{n}}^{\alpha_{n}}=\frac{\partial^{|\alpha|}}{\partial z^{\alpha}}, \quad \partial_{\bar{z}}^{\alpha}=\partial_{\bar{z}_{1}}^{\alpha_{1}} \ldots \partial_{\bar{z}_{n}}^{\alpha_{n}}=\frac{\partial^{|\alpha|}}{\partial \bar{z}^{\alpha}} .
\end{aligned}
$$

Let $X$ be a $C^{\infty}$ orientable paracompact manifold. We let $T X$ and $T^{*} X$ denote the tangent bundle of $X$ and the cotangent bundle of $X$, respectively. The complexified tangent bundle of $X$ and the complexified cotangent bundle of $X$ will be denoted by $\mathbb{C} T X$ and $\mathbb{C} T^{*} X$, respectively. 
We write $\langle\cdot, \cdot\rangle$ to denote the pointwise duality between $T^{*} X$ and $T X$. We extend $\langle\cdot, \cdot\rangle$ bilinearly to $\mathbb{C} T^{*} X \times \mathbb{C} T X$. For $u \in \mathbb{C} T^{*} X, v \in \mathbb{C} T X$, we also write $u(v):=\langle u, v\rangle$.

Let $Y \subset X$ be an open set. The spaces of smooth sections of $E$ over $Y$ and distribution sections of $E$ over $Y$ will be denoted by $C^{\infty}(Y, E)$ and $D^{\prime}(Y, E)$, respectively.

2.2. CR manifolds with $S^{1}$-action. Let $\left(X, T^{1,0} X\right)$ be a compact CR manifold of dimension $2 n+1, n \geq 1$, where $T^{1,0} X$ is a CR structure of $X$. That is, $T^{1,0} X$ is a subbundle of rank $n$ of the complexified tangent bundle $\mathbb{C} T X$, satisfying $T^{1,0} X \cap T^{0,1} X=\{0\}$, where $T^{0,1} X=\overline{T^{1,0} X}$, and $[\mathcal{V}, \mathcal{V}] \subset \mathcal{V}$, where $\mathcal{V}=C^{\infty}\left(X, T^{1,0} X\right)$. We assume that $X$ admits a $S^{1}$ action: $S^{1} \times X \rightarrow X$. We write $e^{i \theta}$ to denote the $S^{1}$ action. Let $T \in C^{\infty}(X, T X)$ be the global real vector field induced by the $S^{1}$ action given by $(T u)(x)=\left.\frac{\partial}{\partial \theta}\left(u\left(e^{i \theta} \circ x\right)\right)\right|_{\theta=0}, u \in C^{\infty}(X)$.

Definition 2.1. We say that the $S^{1}$ action $e^{i \theta}$ is $C R$ if $\left[T, C^{\infty}\left(X, T^{1,0} X\right)\right] \subset C^{\infty}\left(X, T^{1,0} X\right)$ and the $S^{1}$ action is transversal if for each $x \in X, \mathbb{C} T(x) \oplus T_{x}^{1,0} X \oplus T_{x}^{0,1} X=\mathbb{C} T_{x} X$. Moreover, we say that the $S^{1}$ action is locally free if $T \neq 0$ everywhere.

Note that if the $S^{1}$ action is transversal, then it is locally free. We assume throughout that $\left(X, T^{1,0} X\right)$ is a connected CR manifold with a transversal CR $S^{1}$ action $e^{i \theta}$ and we let $T$ be the global vector field induced by the $S^{1}$ action. Let $\omega_{0} \in C^{\infty}\left(X, T^{*} X\right)$ be the global real one form determined by $\left\langle\omega_{0}, u\right\rangle=0$, for every $u \in T^{1,0} X \oplus T^{0,1} X$ and $\left\langle\omega_{0}, T\right\rangle=-1$.

Definition 2.2. For $p \in X$, the Levi form $\mathcal{L}_{p}$ is the Hermitian quadratic form on $T_{p}^{1,0} X$ given by $\mathcal{L}_{p}(U, \bar{V})=-\frac{1}{2 i}\left\langle d \omega_{0}(p), U \wedge \bar{V}\right\rangle, U, V \in T_{p}^{1,0} X$.

Definition 2.3. If the Levi form $\mathcal{L}_{p}$ is positive definite, we say that $X$ is strongly pseudoconvex at $p$. If the Levi form is positive definite at every point of $X$, we say that $X$ is strongly pseudoconvex.

Denote by $T^{* 1,0} X$ and $T^{* 0,1} X$ the dual bundles of $T^{1,0} X$ and $T^{0,1} X$, respectively. Define the vector bundle of $(0, q)$ forms by $T^{* 0, q} X=\Lambda^{q}\left(T^{* 0,1} X\right)$. Put $T^{* 0, \bullet} X:=\oplus_{j \in\{0,1, \ldots, n\}} T^{* 0, j} X$. Let $D \subset X$ be an open subset. Let $\Omega^{0, q}(D)$ denote the space of smooth sections of $T^{* 0, q} X$ over $D$ and let $\Omega_{0}^{0, q}(D)$ be the subspace of $\Omega^{0, q}(D)$ whose elements have compact support in $D$. Put

$$
\begin{aligned}
& \Omega^{0, \bullet}(D):=\oplus_{j \in\{0,1, \ldots, n\}} \Omega^{0, j}(D), \\
& \Omega_{0}^{0, \bullet}(D):=\oplus_{j \in\{0,1, \ldots, n\}} \Omega_{0}^{0, j}(D) .
\end{aligned}
$$

Similarly, if $E$ is a vector bundle over $D$, then we let $\Omega^{0, q}(D, E)$ denote the space of smooth sections of $T^{* 0, q} X \otimes E$ over $D$ and let $\Omega_{0}^{0, q}(D, E)$ be the subspace of $\Omega^{0, q}(D, E)$ whose elements have compact support in $D$. Put

$$
\begin{aligned}
& \Omega^{0, \bullet}(D, E):=\oplus_{j \in\{0,1, \ldots, n\}} \Omega^{0, j}(D, E), \\
& \Omega_{0}^{0, \bullet}(D, E):=\oplus_{j \in\{0,1, \ldots, n\}} \Omega_{0}^{0, j}(D, E) .
\end{aligned}
$$

Fix $\left.\theta_{0} \in\right]-\pi, \pi\left[, \theta_{0}\right.$ small. Let

$$
d e^{i \theta_{0}}: \mathbb{C} T_{x} X \rightarrow \mathbb{C} T_{e^{i \theta_{0}} x} X
$$

denote the differential map of $e^{i \theta_{0}}: X \rightarrow X$. By the CR property of the $S^{1}$ action, we can check that

$$
\begin{gathered}
d e^{i \theta_{0}}: T_{x}^{1,0} X \rightarrow T_{e^{i \theta_{0} x}}^{1,0} X, \\
d e^{i \theta_{0}}: T_{x}^{0,1} X \rightarrow T_{e^{i \theta_{0}} x}^{0,1} X \\
d e^{i \theta_{0}}(T(x))=T\left(e^{i \theta_{0}} x\right) .
\end{gathered}
$$


Let $\left(e^{i \theta_{0}}\right)^{*}: \Lambda^{j}\left(\mathbb{C} T^{*} X\right) \rightarrow \Lambda^{j}\left(\mathbb{C} T^{*} X\right)$ be the pull-back map by $e^{i \theta_{0}}, j=0,1, \ldots, 2 n+1$. From (2.1), it is easy to see that for every $q=0,1, \ldots, n$,

$$
\left(e^{i \theta_{0}}\right)^{*}: T_{e^{i \theta_{0} x}}^{* 0, q} X \rightarrow T_{x}^{* 0, q} X
$$

Let $u \in \Omega^{0, q}(X)$. Define

$$
T u:=\left.\frac{\partial}{\partial \theta}\left(\left(e^{i \theta}\right)^{*} u\right)\right|_{\theta=0} \in \Omega^{0, q}(X) .
$$

For every $\theta \in \mathbb{R}$ and every $u \in C^{\infty}\left(X, \Lambda^{j}\left(\mathbb{C} T^{*} X\right)\right)$, we write $u\left(e^{i \theta} \circ x\right):=\left(e^{i \theta}\right)^{*} u(x)$.

Let $\bar{\partial}_{b}: \Omega^{0, q}(X) \rightarrow \Omega^{0, q+1}(X)$ be the tangential Cauchy-Riemann operator. From the CR property of the $S^{1}$ action, it is straightforward to see that

$$
T \bar{\partial}_{b}=\bar{\partial}_{b} T \text { on } \Omega^{0, \bullet}(X)
$$

Definition 2.4. Let $D \subset U$ be an open set. We say that a function $u \in C^{\infty}(D)$ is rigid if $T u=0$. We say that a function $u \in C^{\infty}(X)$ is Cauchy-Riemann (CR for short) if $\bar{\partial}_{b} u=0$. We call $u$ a rigid $C R$ function if $\bar{\partial}_{b} u=0$ and $T u=0$.

Definition 2.5. Let $F$ be a complex vector bundle over $X$. We say that $F$ is rigid (CR) if $X$ can be covered with open sets $U_{j}$ with trivializing frames $\left\{f_{j}^{1}, f_{j}^{2}, \ldots, f_{j}^{r}\right\}, j=1,2, \ldots$, such that the corresponding transition matrices are rigid $(C R)$. The frames $\left\{f_{j}^{1}, f_{j}^{2}, \ldots, f_{j}^{r}\right\}, j=1,2, \ldots$, are called rigid $(C R)$ frames.

Definition 2.6. Let $F$ be a complex rigid vector bundle over $X$ and let $\langle\cdot \mid \cdot\rangle_{F}$ be a Hermitian metric on $F$. We say that $\langle\cdot \mid \cdot\rangle_{F}$ is a rigid Hermitian metric if for every rigid local frames $f_{1}, \ldots, f_{r}$ of $F$, we have $T\left\langle f_{j} \mid f_{k}\right\rangle_{F}=0$, for every $j, k=1,2, \ldots, r$.

It is known that there is a rigid Hermitian metric on any rigid vector bundle $F$ (see Theorem 2.10 in [5] and Theorem 10.5 in [12]). Note that Baouendi-Rothschild-Treves [2] proved that $T^{1,0} X$ is a rigid complex vector bundle over $X$.

From now on, let $E$ be a rigid $C R$ vector bundle over $X$ and we take a rigid Hermitian metric $\langle\cdot \mid \cdot\rangle_{E}$ on $E$ and take a rigid Hermitian metric $\langle\cdot \mid \cdot\rangle$ on $\mathbb{C} T X$ such that $T^{1,0} X \perp T^{0,1} X$, $T \perp\left(T^{1,0} X \oplus T^{0,1} X\right),\langle T \mid T\rangle=1$. The Hermitian metrics on $\mathbb{C} T X$ and on $E$ induce Hermitian metrics $\langle\cdot \mid \cdot\rangle$ and $\langle\cdot \mid \cdot\rangle_{E}$ on $T^{* 0, \bullet} X$ and $T^{* 0, \bullet} X \otimes E$, respectively. We denote by $d v_{X}=d v_{X}(x)$ the volume form on $X$ induced by the fixed Hermitian metric $\langle\cdot \mid \cdot\rangle$ on $\mathbb{C} T X$. Then we get natural global $L^{2}$ inner products $(\cdot \mid \cdot)_{E},(\cdot \mid \cdot)$ on $\Omega^{0, \bullet}(X, E)$ and $\Omega^{0, \bullet}(X)$, respectively. We denote by $L^{2}\left(X, T^{* 0, q} X \otimes E\right)$ and $L^{2}\left(X, T^{* 0, q} X\right)$ the completions of $\Omega^{0, q}(X, E)$ and $\Omega^{0, q}(X)$ with respect to $(\cdot \mid \cdot)_{E}$ and $(\cdot \mid \cdot)$, respectively. Similarly, we denote by $L^{2}\left(X, T^{* 0, \bullet} X \otimes E\right)$ and $L^{2}\left(X, T^{* 0, \bullet} X\right)$ the completions of $\Omega^{0, \bullet}(X, E)$ and $\Omega^{0, \bullet}(X)$ with respect to $(\cdot \mid \cdot)_{E}$ and $(\cdot \mid \cdot)$, respectively. We extend $(\cdot \mid \cdot)_{E}$ and $(\cdot \mid \cdot)$ to $L^{2}\left(X, T^{* 0, \bullet} X \otimes E\right)$ and $L^{2}\left(X, T^{* 0, \bullet} X\right)$ in the standard way, respectively. For $f \in L^{2}\left(X, T^{* 0, \bullet} X \otimes E\right)$, we denote $\|f\|_{E}^{2}:=(f \mid f)_{E}$. Similarly, for $f \in L^{2}\left(X, T^{* 0, \bullet} X\right)$, we denote $\|f\|^{2}:=(f \mid f)$.

We also write $\bar{\partial}_{b}$ to denote the tangential Cauchy-Riemann operator acting on forms with values in $E$ :

$$
\bar{\partial}_{b}: \Omega^{0, \bullet}(X, E) \rightarrow \Omega^{0, \bullet}(X, E) .
$$

Since $E$ is rigid, we can also define $T u$ for every $u \in \Omega^{0, q}(X, E)$ and we have

$$
T \bar{\partial}_{b}=\bar{\partial}_{b} T \text { on } \Omega^{0, \bullet}(X, E) \text {. }
$$

For every $m \in \mathbb{Z}$, let

$$
\begin{aligned}
& \Omega_{m}^{0, q}(X, E):=\left\{u \in \Omega^{0, q}(X, E) ; T u=i m u\right\}, \quad q=0,1,2, \ldots, n, \\
& \Omega_{m}^{0, \bullet}(X, E):=\left\{u \in \Omega^{0, \bullet}(X, E) ; T u=i m u\right\} .
\end{aligned}
$$


For each $m \in \mathbb{Z}$, we denote by $L_{m}^{2}\left(X, T^{* 0, q} X \otimes E\right)$ and $L_{m}^{2}\left(X, T^{* 0, q} X\right)$ the completions of $\Omega_{m}^{0, q}(X, E)$ and $\Omega_{m}^{0, q}(X)$ with respect to $(\cdot \mid \cdot)_{E}$ and $(\cdot \mid \cdot)$, respectively. Similarly, we denote by $L_{m}^{2}\left(X, T^{* 0, \bullet} X \otimes E\right)$ and $L_{m}^{2}\left(X, T^{* 0, \bullet} X\right)$ the completions of $\Omega_{m}^{0, \bullet}(X, E)$ and $\Omega_{m}^{0, \bullet}(X)$ with respect to $(\cdot \mid \cdot)_{E}$ and $(\cdot \mid \cdot)$, respectively.

2.3. Covering manifolds, Von Neumann dimension. Let $\left(X, T^{1,0} X\right)$ be a compact $\mathrm{CR}$ manifold of dimension $2 n+1, n \geq 1$. Let $\widetilde{X}$ be a paracompact CR manifold, such that there is a discrete group $\Gamma$ acting freely on $\widetilde{X}$ having $X=\widetilde{X} / \Gamma$. Let $\pi: \widetilde{X} \rightarrow X$ be the natural projection with the pull-back map $\pi^{*}: T X \rightarrow T \tilde{X}$. Then $\tilde{X}$ admits a pull-back CR structure $T^{1,0} \widetilde{X}:=\pi^{*} T^{1,0} X$ and, hence, a CR manifold. We assume that $\widetilde{X}$ admits a transversal CR locally free $S^{1}$ action, denoted by $e^{i \theta}$. We further assume that the map

$$
\Gamma \times \widetilde{X} \rightarrow \widetilde{X},(\gamma, \widetilde{x}) \mapsto \gamma \circ \widetilde{x}, \quad \forall \widetilde{x} \in \widetilde{X}, \quad \forall \gamma \in \Gamma .
$$

is $\mathrm{CR}$, i.e.

$$
\gamma_{*}\left(T_{\widetilde{x}}^{1,0} \widetilde{X}\right) \subseteq T_{\gamma \cdot \widetilde{x}}^{1,0} \widetilde{X}
$$

and

$$
e^{i \theta} \circ \gamma \circ \widetilde{x}=\gamma \circ e^{i \theta} \circ \widetilde{x}, \quad \forall \widetilde{x} \in \widetilde{X}, \quad \forall \theta \in[0,2 \pi[, \quad \forall \gamma \in \Gamma .
$$

It is easy to see that the $S^{1}$-action $e^{i \theta}$ on $\tilde{X}$ induces a transversal CR locally free $S^{1}$ action, also denoted by $e^{i \theta}$. We denote by $\widetilde{T}:=\pi^{*} T$ the pull-back one form on $\widetilde{X}$, then $T$ is the global real vector field induced by the $S^{1}$-action on $X$. Let $\widetilde{\omega}_{0}:=\pi^{*} \omega_{0}$ be the pull-back one form on $\widetilde{X}$, where $\omega_{0}$ is the global real one form on $X$ as defined in Subsection 2.2. Then, for $\widetilde{p} \in \widetilde{X}$, the Levi form $\widetilde{\mathcal{L}}_{\widetilde{p}}$ is the Hermitian quadratic form on $T_{\widetilde{p}}^{1,0} \widetilde{X}$ given by

$$
\widetilde{\mathcal{L}}_{\widetilde{p}}(\widetilde{U}, \overline{\widetilde{V}})=-\frac{1}{2 i}\left\langle d \widetilde{\omega}_{0}(\widetilde{p}), \widetilde{U} \wedge \overline{\widetilde{V}}\right\rangle=-\frac{1}{2 i}\left\langle d \omega_{0}(\pi(\widetilde{p})), \pi_{*} \widetilde{U} \wedge \pi_{*} \overline{\widetilde{V}}\right\rangle,
$$

where $\widetilde{U}, \widetilde{V} \in T_{\widetilde{p}}^{1,0} \widetilde{X}$.

As usual, let $\Omega^{0, q}(\widetilde{X})$ denote the space of smooth sections of $\wedge^{q}\left(T^{* 0,1} \widetilde{X}\right)$. We also denote by $\bar{\partial}_{b}: \Omega^{0, q}(\widetilde{X}) \rightarrow \Omega^{0, q+1}(\tilde{X})$ the tangential Cauchy-Riemann operator. Then $\widetilde{T} \bar{\partial}_{b}=$ $\bar{\partial}_{b} \widetilde{T}$ on $\Omega^{0, \bullet}(\widetilde{X})$. Let $E$ be a rigid $\mathrm{CR}$ vector bundle over $X$, then $\widetilde{E}:=\pi^{*} E$ is a $\Gamma$-invariant rigid $\mathrm{CR}$ vector bundle over $\widetilde{X}$. Again let $\Omega^{0, q}(\widetilde{X}, \widetilde{E})$ denote the space of smooth sections of $\wedge^{q}\left(T^{* 0,1} \widetilde{X}\right) \otimes \widetilde{E}$. We again denote by $\bar{\partial}_{b}: \Omega^{0, q}(\widetilde{X}, \widetilde{E}) \rightarrow \Omega^{0, q+1}(\widetilde{X}, \widetilde{E})$ the tangential CauchyRiemann operator. Then again $\widetilde{T} \bar{\partial}_{b}=\bar{\partial}_{b} \widetilde{T}$ on $\Omega^{0, \bullet}(\widetilde{X}, \widetilde{E})$. We denote by $L^{2}\left(\widetilde{X}, T^{* 0, q} \widetilde{X} \otimes \widetilde{E}\right)$ and $L^{2}\left(\widetilde{X}, T^{* 0, q} \widetilde{X}\right)$ the completions of $\Omega^{0, q}(\widetilde{X}, \widetilde{E})$ and $\Omega^{0, q}(\widetilde{X})$ with respect to the corresponding pull-back metrics $(\cdot \mid \cdot)_{\widetilde{E}}$ and $(\cdot \mid \cdot)$. Similarly, we denote by $L^{2}\left(\widetilde{X}, T^{* 0, \bullet} \widetilde{X} \otimes \widetilde{E}\right)$ and $L^{2}\left(\widetilde{X}, T^{* 0, \bullet} \widetilde{X}\right)$ the completions of $\Omega^{0, \bullet}(\widetilde{X}, \widetilde{E})$ and $\Omega^{0, \bullet}(\widetilde{X})$ with respect to the corresponding pull-back metrics $(\cdot \mid \cdot)_{\widetilde{E}}$ and $(\cdot \mid \cdot)$.

As usual, for every $m \in \mathbb{Z}$, let

$$
\begin{aligned}
& \Omega_{m}^{0, q}(\widetilde{X}, \widetilde{E}):=\left\{u \in \Omega^{0, q}(\widetilde{X}, \widetilde{E}) ; \widetilde{T} u=i m u\right\}, \quad q=0,1,2, \ldots, n, \\
& \Omega_{m}^{0, \bullet}(\widetilde{X}, \widetilde{E}):=\left\{u \in \Omega^{0, \bullet}(\widetilde{X}, \widetilde{E}) ; \widetilde{T} u=i m u\right\} .
\end{aligned}
$$

For each $m \in \mathbb{Z}$, we denote by $L_{m}^{2}\left(\widetilde{X}, T^{* 0, q} \widetilde{X} \otimes \widetilde{E}\right)$ and $L_{m}^{2}\left(\widetilde{X}, T^{* 0, q} \widetilde{X}\right)$ the completions of $\Omega_{m}^{0, q}(\widetilde{X}, \widetilde{E})$ and $\Omega_{m}^{0, q}(\widetilde{X})$ with respect to the corresponding pull-back metrics $(\cdot \mid \cdot)_{\widetilde{E}}$ and $(\cdot \mid \cdot)$. Similarly, we denote by $L_{m}^{2}\left(\widetilde{X}, T^{* 0, \bullet} \widetilde{X} \otimes \widetilde{E}\right)$ and $L_{m}^{2}\left(\widetilde{X}, T^{* 0, \bullet} \widetilde{X}\right)$ the completions of $\Omega_{m}^{0, \bullet}(\widetilde{X}, \widetilde{E})$ and $\Omega_{m}^{0, \bullet}(\widetilde{X})$ with respect to the corresponding pull-back metrics $(\cdot \mid \cdot)_{\widetilde{E}}$ and $(\cdot \mid \cdot)$. 
Recall that $U \subset \widetilde{X}$ is called a fundamental domain of the action of $\Gamma$ on $\tilde{X}$ if the following conditions hold:

1. $\tilde{X}=\cup_{\gamma \in \Gamma} \gamma(\bar{U})$,

2. $\gamma_{1}(U) \cap \gamma_{2}(U)=\emptyset$ for $\gamma_{1}, \gamma_{2} \in \Gamma, \gamma_{1} \neq \gamma_{2}$,

3. $\bar{U} \backslash U$ is of measure 0 .

We can take $U$ to be $S^{1}$-invariant and with the pull-back $S^{1}$-action $e^{i \theta}$. We construct such a fundamental domain in the following: From the discussion in the proof of [트, Theorem 2.11], we can find local trivializations $W_{1}, \cdots, W_{N}$ such that $X=\cup_{j=1}^{N} W_{j}$ and each $W_{j}$ is $S^{1}$ invariant. For each $j$, let $\widetilde{W}_{j} \subset \widetilde{X}$ be an $S^{1}$-invariant open set such that $\pi: \widetilde{W}_{j} \rightarrow W_{j}$ is a diffeomorphism and a CR map with inverse $\phi_{j}: W_{j} \rightarrow \widetilde{W}_{j}$. Define $U_{j}=W_{j} \backslash\left(\cup_{i<j} \bar{W}_{i} \cap W_{j}\right)$. Then $U:=\cup_{j} \phi_{j}\left(U_{j}\right)$ is the fundamental domain we want.

It is easy to see that

$$
L^{2}(\widetilde{X}, \widetilde{E}) \simeq L^{2} \Gamma \otimes L^{2}(U, \widetilde{E}) \simeq L^{2} \Gamma \otimes L^{2}(X, E) .
$$

We then have a unitary action of $\Gamma$ by left translations on $L^{2} \Gamma$ by $t_{\gamma} \delta_{\eta}=\delta_{\gamma \eta}$, where $\left\{\delta_{\eta}: \eta \in \Gamma\right\}$ is the orthonormal basis of $L^{2} \Gamma$ formed by the delta functions. It induces a unitary action of $\Gamma$ on $L^{2}(\widetilde{X}, \widetilde{E})$ by $\gamma \mapsto T_{\gamma}=t_{\gamma} \otimes \mathrm{Id}$.

Let us recall the definition of the Von Neumann dimension or $\Gamma$-dimension of a $\Gamma$-module $V \subset L^{2}\left(\widetilde{X}, T^{* 0, q} \widetilde{X} \otimes \widetilde{E}\right)$, see also [19, Definition 3.6.1]. We shall denote by $\mathscr{L}(A)$ the space of bounded operators of the Hilbert space $H$. Let $\mathscr{A}_{\Gamma} \subset \mathscr{L}\left(L^{2} \Gamma\right)$ be the algebra of operators which commute with all left translations and denote the unit element of $\Gamma$ by $e$. We define $\operatorname{Tr}_{\Gamma}[A]:=\left\langle A \delta_{e}, \delta_{e}\right\rangle, A \in \mathscr{A}_{\Gamma}$. Note that a $\Gamma$-module is a left $\Gamma$-invariant subspace $V \subset L^{2} \Gamma$. The orthogonal projection $P_{V}$ on $V$ is in $\mathscr{A}_{\Gamma}$ for a $\Gamma$-module $V$. Set $\operatorname{dim}_{\Gamma} V:=\operatorname{Tr}_{\Gamma}\left[P_{V}\right]$. Now we replace $L^{2} \Gamma$ by $L^{2}\left(\widetilde{X}, T^{* 0, q} \widetilde{X} \otimes \widetilde{E}\right)$. Then to any operator $A \in \mathscr{L}\left(L^{2}\left(\widetilde{X}, T^{* 0, q} \widetilde{X} \otimes \widetilde{E}\right)\right)$, we associate operators $a_{\gamma \eta} \in \mathscr{L}\left(L^{2}\left(U, T^{* 0, q} \widetilde{X} \otimes \widetilde{E}\right)\right)$ such that $a_{\gamma \eta}(f)$ is the projection of $A\left(\delta_{\gamma} \otimes f\right)$ on $\mathbb{C} \delta_{\eta} \otimes L^{2}\left(U, T^{* 0, q} \widetilde{X} \otimes \widetilde{E}\right)$. In addition, if $A \in \mathscr{A}_{\Gamma}$ and $A$ is positive, then $a_{\gamma \eta}=a_{e, \gamma^{-1} \eta}$ and

$$
\operatorname{Tr}_{\Gamma}[A]:=\operatorname{Tr}\left[a_{e e}\right] \geq 0,
$$

is well-defined. The orthogonal projection $P_{V}$ on $V \subset L^{2}\left(\widetilde{X}, T^{* 0, q} \widetilde{X} \otimes \widetilde{E}\right)$ is in $\mathscr{A}_{\Gamma}$ for a $\Gamma$-module $V$.

Definition 2.7. The Von Neumann dimension or $\Gamma$-dimension of a $\Gamma$-module $V$ is defined by

$$
\operatorname{dim}_{\Gamma} V:=\operatorname{Tr}_{\Gamma}\left[P_{V}\right]
$$

\section{Asymptotic expansion of heat kernels of Kohn Laplacians}

In this section, we recall the definition of heat kernels. Then we give a new version of asymptotic expansions of heat kernels of Kohn Laplacians.

3.1. Asymptotics of heat kernels of Kohn Laplacians on a compact CR manifold. Since $T \bar{\partial}_{b}=\bar{\partial}_{b} T$ and $E$ is a rigid CR vector bundle with a rigid Hermitian metric, we have

$$
\bar{\partial}_{b, m}:=\bar{\partial}_{b}: \Omega_{m}^{0, \bullet}(X, E) \rightarrow \Omega_{m}^{0, \bullet}(X, E), \quad \forall m \in \mathbb{Z} .
$$

The $m$-th Fourier component of Kohn-Rossi cohomology is given by

$$
H_{b, m}^{q}(X, E):=\frac{\operatorname{Ker} \bar{\partial}_{b}: \Omega_{m}^{0, q}(X, E) \rightarrow \Omega_{m}^{0, q+1}(X, E)}{\operatorname{Im} \bar{\partial}_{b}: \Omega_{m}^{0, q-1}(X, E) \rightarrow \Omega_{m}^{0, q}(X, E)} .
$$

We also write

$$
\bar{\partial}_{b}^{*}: \Omega^{0, \bullet}(X, E) \rightarrow \Omega^{0, \bullet}(X, E)
$$


to denote the formal adjoint of $\bar{\partial}_{b}$ with respect to $(\cdot \mid \cdot)_{E}$. Since $\langle\cdot \mid \cdot\rangle_{E}$ and $\langle\cdot \mid \cdot\rangle$ are rigid, we can check that

$$
\begin{aligned}
& T \bar{\partial}_{b}^{*}=\bar{\partial}_{b}^{*} T \text { on } \Omega^{0, \bullet}(X, E), \\
& \bar{\partial}_{b, m}^{*}:=\bar{\partial}_{b}^{*}: \Omega_{m}^{0, \bullet}(X, E) \rightarrow \Omega_{m}^{0, \bullet}(X, E), \quad \forall m \in \mathbb{Z} .
\end{aligned}
$$

Now, we fix $m \in \mathbb{Z}$. The $m$-th Fourier component of Kohn Laplacian is given by

$$
\square_{b, m}:=\left(\bar{\partial}_{b, m}+\bar{\partial}_{b, m}^{*}\right)^{2}: \Omega_{m}^{0, \bullet}(X, E) \rightarrow \Omega_{m}^{0, \bullet}(X, E) .
$$

We extend $\square_{b, m}$ to $L_{m}^{2}\left(X, T^{* 0, \bullet} X \otimes E\right)$ by

$$
\square_{b, m}: \operatorname{Dom} \square_{b, m} \subset L_{m}^{2}\left(X, T^{* 0, \bullet} X \otimes E\right) \rightarrow L_{m}^{2}\left(X, T^{* 0, \bullet} X \otimes E\right),
$$

where $\operatorname{Dom} \square_{b, m}:=\left\{u \in L_{m}^{2}\left(X, T^{* 0, \bullet} X \otimes E\right) ; \square_{b, m} u \in L_{m}^{2}\left(X, T^{* 0, \bullet} X \otimes E\right)\right\}$, where for any $u \in L_{m}^{2}\left(X, T^{* 0, \bullet} X \otimes E\right), \square_{b, m} u$ is defined in the sense of distributions. We recall the following results (see Section 3 in [5]).

Theorem 3.1. The Kohn Laplacian $\square_{b, m}$ is self-adjoint, Spec $\square_{b, m}$ is a discrete subset of $[0, \infty[$ and for every $\nu \in \operatorname{Spec} \square_{b, m}, \nu$ is an eigenvalue of $\square_{b, m}$ with finite multiplicity.

For every $\nu \in \operatorname{Spec} \square_{b, m}$, let $\left\{f_{1}^{\nu}, \ldots, f_{d_{\nu}}^{\nu}\right\}$ be an orthonormal frame for the eigenspace of $\square_{b, m}$ with eigenvalue $\nu$. The heat kernel $e^{-t \square_{b, m}}(x, y)$ is given by

$$
e^{-t \square_{b, m}}(x, y)=\sum_{\nu \in \operatorname{Spec} \square_{b, m}} \sum_{j=1}^{d_{\nu}} e^{-\nu t} f_{j}^{\nu}(x) \otimes\left(f_{j}^{\nu}(y)\right)^{\dagger},
$$

where $f_{j}^{\nu}(x) \otimes\left(f_{j}^{\nu}(y)\right)^{\dagger}$ denotes the linear map:

$$
\begin{aligned}
f_{j}^{\nu}(x) \otimes\left(f_{j}^{\nu}(y)\right)^{\dagger}: T_{y}^{* 0, \bullet} X \otimes E_{y} & \rightarrow T_{x}^{* 0, \bullet} X \otimes E_{x}, \\
u(y) & \in T_{y}^{* 0, \bullet} X \otimes E_{y} \rightarrow f_{j}^{\nu}(x)\left\langle u(y) \mid f_{j}^{\nu}(y)\right\rangle_{E} \in T_{x}^{* 0, \bullet} X \otimes E_{x} .
\end{aligned}
$$

Let $e^{-t \square_{b, m}}: L^{2}\left(X, T^{* 0, \bullet} X \otimes E\right) \rightarrow L_{m}^{2}\left(X, T^{* 0, \bullet} X \otimes E\right)$ be the continuous operator with distribution kernel $e^{-t \square_{b, m}}(x, y)$.

We denote by $\dot{\mathcal{R}}$ the Hermitian matrix $\dot{\mathcal{R}} \in \operatorname{End}\left(T^{1,0} X\right)$ such that for $V, W \in T^{1,0} X$,

$$
i d \omega_{0}(V, \bar{W})=\langle\dot{\mathcal{R}} V \mid W\rangle \text {. }
$$

Let $\left\{\omega_{j}\right\}_{j=1}^{n}$ be a local orthonormal frame of $T^{1,0} X$ with dual frame $\left\{\omega^{j}\right\}_{j=1}^{n}$. Set

$$
\gamma_{d}=-i \sum_{l, j=1}^{n} d \omega_{0}\left(\omega_{j}, \bar{\omega}_{l}\right) \bar{\omega}^{l} \wedge \iota_{\bar{\omega}_{j}}
$$

where $\iota_{\bar{\omega}_{j}}$ denotes the interior product of $\bar{\omega}_{j}$. Then $\gamma_{d} \in \operatorname{End}\left(T^{* 0, \bullet} X\right)$ and $-i d \omega_{0}$ acts as the derivative $\gamma_{d}$ on $T^{* 0, \bullet} X$. If we choose $\left\{\omega_{j}\right\}_{j=1}^{n}$ to be an orthonormal basis of $T^{1,0} X$ such that

$$
\dot{\mathcal{R}}(x)=\operatorname{diag}\left(a_{1}(x), \cdots, a_{n}(x)\right) \in \operatorname{End}\left(T_{x}^{1,0} X\right),
$$

then

$$
\gamma_{d}(x)=-\sum_{j=1}^{n} a_{j}(x) \bar{\omega}^{j} \wedge \iota_{\bar{\omega}_{j}} .
$$

Define $\operatorname{det} \dot{\mathcal{R}}(x):=a_{1}(x) \cdots a_{n}(x)$.

Fix $x, y \in X$. Let $d(x, y)$ denote the standard Riemannian distance of $x$ and $y$ with respect to the given Hermitian metric. Take $\zeta$

$$
o<\zeta<\inf \left\{\frac{2 \pi}{p_{k}},\left|\frac{2 \pi}{p_{r}}-\frac{2 \pi}{p_{r+1}}\right|, r=1, \cdots, k-1\right\} .
$$


For $x \in X$, put

$$
\hat{d}\left(x, X_{\text {sing }}\right):=\inf \left\{d\left(x, e^{-i \theta} x\right) ; \zeta \leq \theta \leq \frac{2 \pi}{p}-\zeta\right\} .
$$

The following result generalizes Theorem 3.1 in [13].

Theorem 3.2. With the above notations and assumptions, for every $\epsilon>0$, there are $m_{0}>0$, $\varepsilon_{0}>0$ and $C>0$ such that for all $m \geq m_{0}$, we have

$$
\begin{aligned}
& \left|e^{-\frac{t}{m} \square_{b, m}}(x, x)-\sum_{s=1}^{p} e^{\frac{2 \pi(s-1)}{p} m i}(2 \pi)^{-n-1} m^{n} \frac{\operatorname{det}(\dot{\mathcal{R}}) \exp \left(t \gamma_{d}\right)}{\operatorname{det}(1-\exp (-t \dot{\mathcal{R}}))}(x) \otimes \operatorname{Id}_{E_{x}}\right| \\
& \leq \epsilon m^{n}+C m^{n} t^{-n} e^{\frac{-\varepsilon_{0} m \hat{d}\left(x, X_{\text {sing }}\right)^{2}}{t}}, \quad \forall(t, x) \in \mathbb{R}_{+} \times X_{\text {reg }} .
\end{aligned}
$$

Proof. We use the notations from Section 3 in [13]. Recall that $\Gamma_{m}$ is defined in [13, (3.31)] (see also (3.29) ). For $x \in X_{\text {reg }}$, we have

$$
\begin{aligned}
\Gamma_{m}(t, x, x)= & \frac{1}{2 \pi} \sum_{j=1}^{N} \int_{0}^{2 \pi} H_{j, m}\left(t, x, e^{i u} \circ x\right) e^{i m u} d u \\
= & \frac{1}{2 \pi} \sum_{s=1}^{p} e^{\frac{2 \pi(s-1)}{p} m i} \sum_{j=1}^{N} \int_{0}^{\frac{2 \pi}{p}} H_{j, m}\left(t, x, e^{i u} \circ x\right) e^{i m u} d u \\
= & \frac{1}{2 \pi} \sum_{s=1}^{p} e^{\frac{2 \pi(s-1)}{p} m i} \sum_{j=1}^{N} \int_{u \in\left[\zeta, \frac{2 \pi}{p}-\zeta\right]} H_{j, m}\left(t, x, e^{i u} \circ x\right) e^{i m u} d u \\
& +\frac{1}{2 \pi} \sum_{s=1}^{p} e^{\frac{2 \pi(s-1)}{p} m i} \sum_{j=1}^{N} \int_{-\zeta}^{\zeta} H_{j, m}\left(t, x, e^{i u} \circ x\right) e^{i m u} d u,
\end{aligned}
$$

where $H_{j, m}$ is defined in [13, (3.30)] (see also (3.29)). From [13, (3.29), (3.34)] and [5, (6.4)], there are $\varepsilon_{0}>0$ and $C_{0}$ independent of $j, x, m, t$ such that, for all $t \in \mathbb{R}_{+}$and for all $m \in \mathbb{N}$, we have

$$
\left|\frac{1}{2 \pi} \int_{u \in\left[\zeta, \frac{2 \pi}{p}-\zeta\right]} H_{j, m}\left(t, x, e^{i u} \circ x\right) e^{i m u} d u\right| \leq C_{0} m^{n} t^{-n} e^{\frac{-\varepsilon_{0} m \hat{d}\left(x, X_{\text {sing }}\right)^{2}}{t}} .
$$

Then the proof is completed by applying [13, (3.32), (3.39)] and (3.12).

Remark 3.3. It is easy to check that

$$
\sum_{s=1}^{p} e^{\frac{2 \pi(s-1)}{p} m i}= \begin{cases}p & p \mid m \\ 0 & p \nmid m .\end{cases}
$$

3.2. BRT trivializations. To prove Theorem [3.2, we need some preparations. We first need the following result due to Baouendi-Rothschild-Treves [2].

Theorem 3.4. For every point $x_{0} \in X$, we can find local coordinates $x=\left(x_{1}, \cdots, x_{2 n+1}\right)=$ $(z, \theta)=\left(z_{1}, \cdots, z_{n}, \theta\right), z_{j}=x_{2 j-1}+i x_{2 j}, j=1, \cdots, n, x_{2 n+1}=\theta$, defined in some small neighborhood $D=\left\{(z, \theta):|z|<\delta,-\varepsilon_{0}<\theta<\varepsilon_{0}\right\}$ of $x_{0}, \delta>0,0<\varepsilon_{0}<\pi$, such that $\left(z\left(x_{0}\right), \theta\left(x_{0}\right)\right)=(0,0)$ and

$$
\begin{aligned}
& T=\frac{\partial}{\partial \theta} \\
& Z_{j}=\frac{\partial}{\partial z_{j}}+i \frac{\partial \varphi}{\partial z_{j}}(z) \frac{\partial}{\partial \theta}, j=1, \cdots, n
\end{aligned}
$$


where $Z_{j}(x), j=1, \cdots, n$, form a basis of $T_{x}^{1,0} X$, for each $x \in D$, and $\varphi(z) \in C^{\infty}(D, \mathbb{R})$ is independent of $\theta$. We call $(D,(z, \theta), \varphi)$ BRT trivialization.

By using BRT trivialization, we get another way to define $T u, \forall u \in \Omega^{0, q}(X)$. Let $(D,(z, \theta), \varphi)$ be a BRT trivialization. It is clear that

$$
\left\{d \overline{z_{j_{1}}} \wedge \cdots \wedge d \overline{z_{j_{q}}}, 1 \leq j_{1}<\cdots<j_{q} \leq n\right\}
$$

is a basis for $T_{x}^{* 0, q} X$, for every $x \in D$. Let $u \in \Omega^{0, q}(X)$. On $D$, we write

$$
u=\sum_{1 \leq j_{1}<\cdots<j_{q} \leq n} u_{j_{1} \cdots j_{q}} d \overline{z_{j_{1}}} \wedge \cdots \wedge d \overline{z_{j_{q}}} \text {. }
$$

Then, on $D$, we can check that

$$
T u=\sum_{1 \leq j_{1}<\cdots<j_{q} \leq n}\left(T u_{j_{1} \cdots j_{q}}\right) d \overline{z_{j_{1}}} \wedge \cdots \wedge d \overline{z_{j_{q}}}
$$

and $T u$ is independent of the choice of BRT trivializations. Note that, on BRT trivialization $(D,(z, \theta), \varphi)$, we have

$$
\bar{\partial}_{b}=\sum_{j=1}^{n} d \bar{z}_{j} \wedge\left(\frac{\partial}{\partial \bar{z}_{j}}-i \frac{\partial \varphi}{\partial \bar{z}_{j}}(z) \frac{\partial}{\partial \theta}\right) .
$$

3.3. Local heat kernels on BRT trivializations. Until further notice, we fix $m \in \mathbb{Z}$. Let $B:=(D,(z, \theta), \varphi)$ be a BRT trivialization. We may assume that $D=U \times]-\varepsilon, \varepsilon[$, where $\varepsilon>0$ and $U$ is an open set of $\mathbb{C}^{n}$. Since $E$ is rigid, we can consider $E$ as a holomorphic vector bundle over $U$. We may assume that $E$ is trivial on $U$. Consider a trivial line bundle $L \rightarrow U$ with non-trivial Hermitian fiber metric $|1|_{h^{L}}^{2}=e^{-2 \varphi}$. Let $\left(L^{m}, h^{L^{m}}\right) \rightarrow U$ be the $m$-th power of $\left(L, h^{L}\right)$. Let $\Omega^{0, q}\left(U, E \otimes L^{m}\right)$ and $\Omega^{0, q}(U, E)$ be the spaces of $(0, q)$ forms on $U$ with values in $E \otimes L^{m}$ and $E$, respectively, $q=0,1,2, \ldots, n$. Put

$$
\begin{aligned}
& \Omega^{0, \bullet}\left(U, E \otimes L^{m}\right):=\oplus_{j \in\{0,1, \ldots, n\}} \Omega^{0, j}\left(U, E \otimes L^{m}\right), \\
& \Omega^{0, \bullet}(U, E):=\oplus_{j \in\{0,1, \ldots, n\}} \Omega^{0, j}(U, E) .
\end{aligned}
$$

Since $L$ is trivial, from now on, we identify $\Omega^{0, \bullet}(U, E)$ with $\Omega^{0, \bullet}\left(U, E \otimes L^{m}\right)$. Since the Hermitian fiber metric $\langle\cdot \mid \cdot\rangle_{E}$ is rigid, we can consider $\langle\cdot \mid \cdot\rangle_{E}$ as a Hermitian fiber metric on the holomorphic vector bundle $E$ over $U$. Let $\langle\cdot, \cdot\rangle$ be the Hermitian metric on $\mathbb{C} T U$ given by

$$
\left\langle\frac{\partial}{\partial z_{j}}, \frac{\partial}{\partial z_{k}}\right\rangle=\left\langle\frac{\partial}{\partial z_{j}}+i \frac{\partial \varphi}{\partial z_{j}}(z) \frac{\partial}{\partial \theta} \mid \frac{\partial}{\partial z_{k}}+i \frac{\partial \varphi}{\partial z_{k}}(z) \frac{\partial}{\partial \theta}\right\rangle, \quad j, k=1,2, \ldots, n .
$$

$\langle\cdot, \cdot\rangle$ induces a Hermitian metric on $T^{* 0, \bullet} U:=\oplus_{j=0}^{n} T^{* 0, j} U$, where $T^{* 0, j} U$ is the bundle of $(0, j)$ forms on $U, j=0,1, \ldots, n$. We shall also denote this induced Hermitian metric on $T^{* 0, \bullet} U$ by $\langle\cdot, \cdot\rangle$. The Hermitian metrics on $T^{* 0, \bullet} U$ and $E$ induce a Hermitian metric on $T^{* 0, \bullet} U \otimes E$. We shall also denote this induced metric by $\langle\cdot \mid \cdot\rangle_{E}$. Let $(\cdot, \cdot)$ be the $L^{2}$ inner product on $\Omega^{0, \bullet}(U, E)$ induced by $\langle\cdot, \cdot\rangle,\langle\cdot \mid \cdot\rangle_{E}$. Similarly, let $(\cdot, \cdot)_{m}$ be the $L^{2}$ inner product on $\Omega^{0, \bullet}\left(U, E \otimes L^{m}\right)$ induced by $\langle\cdot, \cdot\rangle,\langle\cdot \mid \cdot\rangle_{E}$ and $h^{L^{m}}$.

The curvature of $L$ induced by $h^{L}$ is given by $R^{L}:=2 \partial \bar{\partial} \varphi$. Let $\dot{R}^{L} \in \operatorname{End}\left(T^{1,0} U\right)$ be the Hermitian matrix given by

$$
R^{L}(W, \bar{Y})=\left\langle\dot{R}^{L} W, Y\right\rangle, \quad W, Y \in T^{1,0} U .
$$

Let $\left\{w_{j}\right\}_{j=1}^{n}$ be a local orthonormal frame of $T^{1,0} U$ with dual frame $\left\{w^{j}\right\}_{j=1}^{n}$. Set

$$
\omega_{d}=-\sum_{l, j} R^{L}\left(w_{j}, \bar{w}_{l}\right) \bar{w}^{l} \wedge \iota_{\bar{w}_{j}},
$$

where $\iota_{\bar{w}_{j}}$ denotes the interior product of $\bar{w}_{j}$. 
Let

$$
\bar{\partial}: \Omega^{0, \bullet}\left(U, E \otimes L^{m}\right) \rightarrow \Omega^{0, \bullet}\left(U, E \otimes L^{m}\right)
$$

be the Cauchy-Riemann operator and let

$$
\bar{\partial}^{*, m}: \Omega^{0, \bullet}\left(U, E \otimes L^{m}\right) \rightarrow \Omega^{0, \bullet}\left(U, E \otimes L^{m}\right)
$$

be the formal adjoint of $\bar{\partial}$ with respect to $(\cdot, \cdot)_{m}$. Put

$$
\square_{B, m}:=\left(\bar{\partial}+\bar{\partial}^{*, m}\right)^{2}: \Omega^{0, \bullet}\left(U, E \otimes L^{m}\right) \rightarrow \Omega^{0, \bullet}\left(U, E \otimes L^{m}\right) .
$$

We need the following result (see Lemma 5.1 in [5])

Lemma 3.5. Let $u \in \Omega_{m}^{0, \bullet}(X, E)$. On D, we write $u(z, \theta)=e^{i m \theta} \widetilde{u}(z), \widetilde{u}(z) \in \Omega^{0, \bullet}(U, E)$. Then,

$$
e^{-m \varphi} \square_{B, m}\left(e^{m \varphi} \widetilde{u}\right)=e^{-i m \theta} \square_{b, m}(u) .
$$

Let $z, w \in U$ and let $T(z, w) \in\left(T_{w}^{* 0, \bullet} U \otimes E_{w}\right) \otimes\left(T_{z}^{* 0, \bullet} U \otimes E_{z}\right)$. We write $|T(z, w)|$ to denote the standard pointwise matrix norm of $T(z, w)$ induced by $\langle\cdot \mid \cdot\rangle_{E}$. Let $\Omega_{0}^{0, \bullet}(U, E)$ be the subspace of $\Omega^{0, \bullet}(U, E)$ whose elements have compact support in $U$. Let $d v_{U}$ be the volume form on $U$ induced by $\langle\cdot, \cdot\rangle$. Assume $T(z, w) \in C^{\infty}\left(U \times U,\left(T_{w}^{* 0, \bullet} U \otimes E_{w}\right) \otimes\left(T_{z}^{* 0, \bullet} \otimes \otimes E_{z}\right)\right)$. Let $u \in \Omega_{0}^{0, \bullet}(U, E)$. We define the integral $\int T(z, w) u(w) d v_{U}(w)$ in the standard way. Let $G(t, z, w) \in C^{\infty}\left(\mathbb{R}_{+} \times U \times U,\left(T_{w}^{* 0, \bullet} U \otimes E_{w}\right) \otimes\left(T_{z}^{* 0, \bullet} U \otimes E_{z}\right)\right)$. We write $G(t)$ to denote the continuous operator

$$
\begin{aligned}
G(t): \Omega_{0}^{0, \bullet}(U, E) & \rightarrow \Omega^{0, \bullet}(U, E), \\
u & \rightarrow \int G(t, z, w) u(w) d v_{U}(w)
\end{aligned}
$$

and we write $G^{\prime}(t)$ to denote the continuous operator

$$
\begin{aligned}
G^{\prime}(t): \Omega_{0}^{0, \bullet}(U, E) & \rightarrow \Omega^{0, \bullet}(U, E), \\
u & \rightarrow \int \frac{\partial G(t, z, w)}{\partial t} u(w) d v_{U}(w) .
\end{aligned}
$$

We consider the heat operator of $\square_{B, m}$. By using the standard Dirichlet heat kernel construction (see [10]) and the proofs of Theorem 1.6.1 and Theorem 5.5.9 in [19], we deduce the following

Theorem 3.6. There is $A_{B, m}(t, z, w) \in C^{\infty}\left(\mathbb{R}_{+} \times U \times U,\left(T_{w}^{* 0, \bullet} U \otimes E_{w}\right) \otimes\left(T_{z}^{* 0, \bullet} U \otimes E_{z}\right)\right)$ such that

$$
\begin{aligned}
& \lim _{t \rightarrow 0+} A_{B, m}(t)=I \text { in } D^{\prime}\left(U, T^{* 0, \bullet} U \otimes E\right), \\
& A_{B, m}^{\prime}(t) u+\frac{1}{m} A_{B, m}(t)\left(\square_{B, m} u\right)=0, \quad \forall u \in \Omega_{0}^{0, \bullet}(U, E), \quad \forall t>0,
\end{aligned}
$$

and $A_{B, m}(t, z, w)$ satisfies the following:

(I) For every compact set $K \Subset U, \alpha_{1}, \alpha_{2}, \beta_{1}, \beta_{2} \in \mathbb{N}_{0}^{n}$, there are constants $C_{\alpha_{1}, \alpha_{2}, \beta_{1}, \beta_{2}, K}>0$ and $\varepsilon_{0}>0$ independent of $t$ and $m$ such that

$$
\begin{aligned}
& \left|\partial_{z}^{\alpha_{1}} \partial_{\bar{z}}^{\alpha_{2}} \partial_{w}^{\beta_{1}} \partial_{\bar{w}}^{\beta_{2}}\left(A_{B, m}(t, z, w) e^{m(\varphi(w)-\varphi(z))}\right)\right| \\
& \leq C_{\alpha_{1}, \alpha_{2}, \beta_{1}, \beta_{2}, K}\left(\frac{m}{t}\right)^{n+\left|\alpha_{1}\right|+\left|\alpha_{2}\right|+\left|\beta_{1}\right|+\left|\beta_{2}\right|} e^{-m \varepsilon_{0} \frac{|z-w|^{2}}{t}}, \quad \forall(t, z, w) \in \mathbb{R}_{+} \times K \times K .
\end{aligned}
$$

(II) $A_{B, m}(t, z, z)$ admits an asymptotic expansion:

$$
A_{B, m}(t, z, z)=(2 \pi)^{-n} m^{n} \frac{\operatorname{det}\left(\dot{R}^{L}\right) \exp \left(t \omega_{d}\right)}{\operatorname{det}\left(1-\exp \left(-t \dot{R}^{L}\right)\right)}(z) \otimes \operatorname{Id}_{E_{z}}+o\left(m^{n}\right)
$$


in $C^{\ell}\left(U, \operatorname{End}\left(T^{* 0, \bullet} U\right) \otimes E\right)$ locally uniformly on $\mathbb{R}_{+} \times U$, for every $\ell \in \mathbb{N}$. Here we use the convention that if an eigenvalue $a_{j}(z)$ of $\dot{R}^{L}(z)$ is zero, then its contribution for $\frac{\operatorname{det}\left(\dot{R}^{L}\right)}{\operatorname{det}\left(1-\exp \left(-t \dot{R}^{L}\right)\right)}(z)$ is $\frac{1}{t}$.

\section{4. $L^{2}$ Kohn-Rossi cohomology on a covering manifold. Let}

$$
\widetilde{\square}_{b}: \operatorname{Dom} \widetilde{\square}_{b} \subset L^{2}\left(\widetilde{X}, T^{* 0, \bullet} \widetilde{X}\right) \rightarrow L^{2}\left(\widetilde{X}, T^{* 0, \bullet} \widetilde{X}\right)
$$

be the Gaffney extension of the pull-back Kohn Laplacian on $\tilde{X}$. By a result of Gaffney, $\widetilde{\square}_{b}$ is a positive self-adjoint operator (see Proposition 3.1.2 in Ma-Marinescu [19]). That is, $\widetilde{\square}_{b}$ is self-adjoint and the spectrum of $\widetilde{\square}_{b}$ is contained in $\overline{\mathbb{R}}_{+}$. Now, we fix $m \in \mathbb{Z}$. As in (3.3), we introduce the $m$-th Fourier component of the Kohn Laplacian $\widetilde{\square}_{b, m}$ on $\Omega_{m}^{0, \bullet}(\widetilde{X}, \widetilde{E})$. We can easily see that $\widetilde{\square}_{b, m}$ is also self-adjoint. By the second isomorphism of (2.10), we can see that, for any $\gamma \in \Gamma$,

$$
T_{\gamma}\left(\operatorname{Dom}\left(\widetilde{\square}_{b, m}\right)\right) \subset \operatorname{Dom}\left(\widetilde{\square}_{b, m}\right), \quad T_{\gamma} \widetilde{\square}_{b, m}=\widetilde{\square}_{b, m} T_{\gamma} \quad \text { on } \quad \operatorname{Dom}\left(\widetilde{\square}_{b, m}\right) .
$$

Consider the spectral resolution $E_{\lambda}^{q}\left(\widetilde{\square}_{b, m}\right)$ of $\widetilde{\square}_{b, m}$ acting on $L_{m}^{2}\left(\widetilde{X}, T^{* 0, q} \widetilde{X} \otimes \widetilde{E}\right)$. (See [19, Appendix C.2]). The proof of the following lemma is similar to Lemma 3.6.3 in Ma-Marinescu [19].

Lemma 3.7. For any $q=0,1, \cdots, n$ and $\lambda \in \mathbb{R}$, then $E_{\lambda}^{q}\left(\widetilde{\square}_{b, m}\right)$ commutes with $\Gamma$, its Schwartz kernel is smooth and

$$
\operatorname{dim}_{\Gamma} E_{\lambda}^{q}\left(\widetilde{\square}_{b, m}\right)<+\infty .
$$

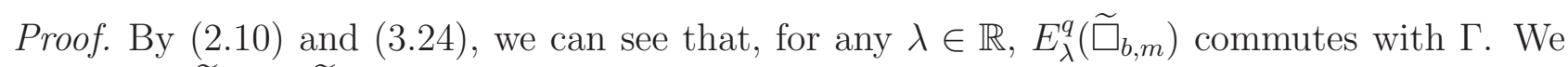
claim that $\widetilde{\square}_{b, m}-\widetilde{T}^{2} \equiv \Delta$ is a second order elliptic operator, so is $\Delta-m^{2}$. Its principal symbol is locally written as

$$
\sigma_{\Delta}(\widetilde{x}, \xi)=\sigma_{\widetilde{\square}_{b, m}}(\widetilde{x}, \xi)-\sigma_{\widetilde{T}^{2}}(\widetilde{x}, \xi)=\sum_{j=1}^{n}\left|\sigma_{L_{j}}(\widetilde{x}, \xi)\right|^{2}-\sigma_{\widetilde{T}}(\widetilde{x}, \xi)^{2},
$$

where $\xi=\left(\xi_{1}, \ldots, \xi_{2 n}, \xi_{2 n+1}\right)$ and $\left\{L_{j}\right\}$ is an orthonormal basis of $T_{x}^{0,1} \widetilde{X}$. It is well-known that the characteristic manifold of $\widetilde{\square}_{b}$ is

$$
\Sigma=\left\{\left(\widetilde{x}, c \widetilde{\omega}_{0}(\widetilde{x})\right) \in T^{*} \widetilde{X}: c \neq 0\right\} .
$$

It means that $\sigma_{\widetilde{\square}_{b, m}}(\widetilde{x}, \xi)>0$ if and only if $\left(\xi_{1}, \ldots, \xi_{2 n}\right) \neq 0$. Meanwhile, in a local BRT coordinate [2], we have $\widetilde{T}=\frac{\partial}{\partial \theta}$, then $\sigma_{\widetilde{T}}=i \xi_{2 n+1}$. That is, $\sigma_{\widetilde{T}^{2}}=-\xi_{2 n+1}^{2}$. Then the claim is proved. By the spectral theorem, cf. [19, Theorem C.2.1], we have $\operatorname{Im}\left(E_{\lambda}\left(\Delta-m^{2}\right)\right) \subset$ $\operatorname{Dom}\left(\left(\Delta-m^{2}\right)^{k}\right)$ for $k \in \mathbb{N}$. Using the uniform Sobolev spaces [23, pp. 511-512], it is easy to see that $\operatorname{Im}\left(E_{\lambda}\left(\Delta-m^{2}\right)\right) \subset \Omega^{\bullet}(\widetilde{X}, \widetilde{E})$, so that $E_{\lambda}\left(\Delta-m^{2}\right): L^{2}\left(\widetilde{X}, T^{* 0}, \widetilde{X} \otimes \widetilde{E}\right) \rightarrow \Omega^{\bullet}(\widetilde{X}, \widetilde{E})$ is linear continuous. Hence, $\operatorname{Im} E_{\lambda}\left(\widetilde{\square}_{b, m}\right)=\operatorname{Im}\left(E_{\lambda}\left(\Delta-m^{2}\right)\right) \cap L_{m}^{2}\left(\widetilde{X}, T^{* 0, \bullet} \widetilde{X} \otimes \widetilde{E}\right) \subset \Omega^{\bullet}(\widetilde{X}, \widetilde{E}) \cap$ $L_{m}^{2}\left(\widetilde{X}, T^{* 0, \bullet} \widetilde{X} \otimes \widetilde{E}\right)=\Omega_{m}^{\bullet}(\widetilde{X}, \widetilde{E})$ and $E_{\lambda}\left(\widetilde{\square}_{b, m}\right): L_{m}^{2}\left(\widetilde{X}, T^{* 0, \bullet} \widetilde{X} \otimes \widetilde{E}\right) \rightarrow \Omega_{m}^{\bullet}(\widetilde{X}, \widetilde{E})$ is also linear continuous. By Schwartz kernel theorem, the kernel $E_{\lambda}\left(\widetilde{\square}_{b, m}\right)(\widetilde{x}, \widetilde{x})$ of $E_{\lambda}\left(\widetilde{\square}_{b, m}\right)$ with respect to $d v_{\widetilde{X}}(\widetilde{x})$ is smooth. By [19, (3.6.12)],

$$
\operatorname{dim}_{\Gamma} E_{\lambda}\left(\widetilde{\square}_{b, m}\right)=\int_{U} \operatorname{Tr}\left[E_{\lambda}\left(\widetilde{\square}_{b, m}\right)(\widetilde{x}, \widetilde{x})\right] d v_{\widetilde{X}}(\widetilde{x})<+\infty .
$$

Definition 3.8. (a) The $m$-th Fourier component of the space of harmonic forms $\mathcal{H} \bullet(\widetilde{X}, \widetilde{E})$ is defined by

$$
\mathcal{H}_{b, m}^{\bullet}(\widetilde{X}, \widetilde{E}):=\operatorname{Ker}\left(\widetilde{\square}_{b, m}\right)=\left\{s \in \operatorname{Dom} \widetilde{\square}_{b, m}: \widetilde{\square}_{b, m} s=0\right\} .
$$


(b) The m-th Fourier component of the q-th reduced $L^{2}$ Kohn-Rossi cohomology is given by

$$
\bar{H}_{b,(2), m}^{q}(\widetilde{X}, \widetilde{E}):=\frac{\operatorname{Ker} \bar{\partial}_{b} \cap L_{m}^{2}\left(\widetilde{X}, T^{* 0, q} \widetilde{X} \otimes \widetilde{E}\right)}{\left[\operatorname{Im} \bar{\partial}_{b} \cap L_{m}^{2}\left(\widetilde{X}, T^{* 0, q} \widetilde{X} \otimes \widetilde{E}\right)\right]},
$$

where $[V]$ denotes the closure of the space $V$.

We can easily obtain the following weak Hodge decomposition

$$
L_{m}^{2}\left(\widetilde{X}, T^{* 0, \bullet} \widetilde{X} \otimes \widetilde{E}\right)=\mathcal{H}^{\bullet}(\widetilde{X}, \widetilde{E}) \oplus\left[\operatorname{Im}\left(\bar{\partial}_{b, m}\right)\right] \oplus\left[\operatorname{Im}\left(\bar{\partial}_{b, m}^{*}\right)\right]
$$

By (3.26), we the the isomorphism

$$
\bar{H}_{b,(2), m}^{\bullet}(\widetilde{X}, \widetilde{E}) \cong \mathcal{H}_{b}^{\bullet}(\widetilde{X}, \widetilde{E}) .
$$

3.5. Asymptotics of heat kernels of Kohn Laplacians on a covering manifold. Assume that $X=D_{1} \cup D_{2} \cup \cdots \cup D_{N}$, where $B_{j}:=\left(D_{j},(z, \theta), \varphi_{j}\right)$ is a BRT trivialization, for each $j$. We may assume that, for each $\left.j, D_{j}=U_{j} \times\right]-2 \delta_{j}, 2 \widetilde{\delta}_{j}\left[\subset \mathbb{C}^{n} \times \mathbb{R}, \delta_{j}>0, \widetilde{\delta}_{j}>0, U_{j}=\right.$ $\left\{z \in \mathbb{C}^{n} ;|z|<l_{j}\right\}$. For each $j$, put $\left.\hat{D}_{j}=\hat{U}_{j} \times\right]-\frac{\delta_{j}}{2}$, $\frac{\widetilde{\delta}_{j}}{2}\left[\right.$, where $\hat{U}_{j}=\left\{z \in \mathbb{C}^{n} ;|z|<\frac{l_{j}}{2}\right\}$. We may suppose that $X=\hat{D}_{1} \cup \hat{D}_{2} \cup \cdots \cup \hat{D}_{N}$.

Let $\left\{\psi_{j}\right\}$ be a partition of unity subordinate to $\left\{\hat{D}_{j}\right\}$. Then $\left\{\widetilde{\psi}_{\gamma, j}:=\psi_{i} \circ \pi\right\}$ is a partition of unity subordinate to $\left\{\widetilde{D}_{\gamma, j}\right\}$, where $\pi^{-1}\left(\hat{D}_{j}\right)=\cup_{\gamma \in \Gamma} \widetilde{D}_{\gamma, j}$ and $\widetilde{D}_{\gamma_{1}, j}$ and $\widetilde{D}_{\gamma_{2}, j}$ are disjoint for $\gamma_{1} \neq \gamma_{2}$. For each $\gamma \in \Gamma$ and each $j$, we have $\left.\widetilde{D}_{\gamma, j}=\widetilde{U}_{\gamma, j} \times\right]-\frac{\delta_{\gamma, j}}{2}, \frac{\widetilde{\delta}_{\gamma, j}}{2}\left[\right.$, where $\widetilde{U}_{\gamma, j}=$ $\left\{z \in \mathbb{C}^{n} ;|z|<\frac{l_{\gamma, j}}{2}\right\}$. Then $\tilde{X}=\bigcup_{\gamma \in \Gamma} \bigcup_{j=1}^{N} \widetilde{D}_{\gamma, j}$.

Fix $\gamma \in \Gamma$ and $j=1,2, \ldots, N$. Put

$$
K_{\gamma, j}=\left\{z \in \widetilde{U}_{\gamma, j} \text {; there is a } \theta \in\right]-\frac{\delta_{\gamma, j}}{2}, \frac{\widetilde{\widetilde{\gamma}}_{\gamma, j}}{2}\left[\text { such that } \widetilde{\psi}_{\gamma, j}(z, \theta) \neq 0\right\} .
$$

Let $\tau_{\gamma, j}(z) \in C_{0}^{\infty}\left(\widetilde{U}_{\gamma, j}\right)$ with $\tau_{\gamma, j} \equiv 1$ on some neighborhood $W_{\gamma, j}$ of $K_{\gamma, j}$. Let $\sigma_{\gamma, j} \in C_{0}^{\infty}(]-$ $\frac{\delta_{\gamma, j}}{2}, \frac{\widetilde{\gamma}_{\gamma, j}}{2}[)$ with $\int \sigma_{\gamma, j}(\theta) d \theta=1$. Let $\widetilde{A}_{B_{\gamma, j}, m}(t, z, w) \in C^{\infty}\left(\mathbb{R}_{+} \times \widetilde{U}_{\gamma, j} \times \widetilde{U}_{\gamma, j},\left(T_{w}^{* 0, \bullet} \widetilde{U}_{\gamma, j} \otimes \widetilde{E}_{w}\right) \otimes\right.$ $\left.\left(T_{z}^{* 0,} \widetilde{U}_{\gamma, j} \otimes \widetilde{E}_{z}\right)\right)$ be as in Theorem 3.6 ,

Put

$$
\widetilde{H}_{\gamma, j, m}(t, \widetilde{x}, \widetilde{y})=\widetilde{\psi}_{\gamma, j}(\widetilde{x}) e^{-m \varphi_{j}(z)+i m \theta} \widetilde{A}_{B_{\gamma, j}, m}(t, z, w) e^{m \varphi_{\gamma, j}(w)-i m \eta} \tau_{\gamma, j}(w) \sigma_{\gamma, j}(\eta),
$$

where $\widetilde{x}=(z, \theta), \widetilde{y}=(w, \eta) \in \mathbb{C}^{n} \times \mathbb{R}$. Let

$$
\widetilde{\Gamma}_{m}(t, \widetilde{x}, \widetilde{y}):=\frac{1}{2 \pi} \sum_{\gamma \in \Gamma} \sum_{j=1}^{N} \int_{-\pi}^{\pi} \widetilde{H}_{\gamma, j, m}\left(t, \widetilde{x}, e^{i u} \circ \widetilde{y}\right) e^{i m u} d u
$$

Note that when $\Gamma=\{e\}, \widetilde{\Gamma}_{m}(t, \widetilde{x}, \widetilde{y})=\Gamma_{m}(t, \pi(\widetilde{x}), \pi(\widetilde{y}))$ is defined in [13, (3.31)].

From Lemma 3.5, off-diagonal estimates of $\widetilde{A}_{B_{j}, m}(t, \widetilde{x}, \widetilde{y})$ (see (3.22)), we can repeat the proof of Theorem 5.14 in [5] with minor change and deduce that

Theorem 3.9. For every $\ell \in \mathbb{N}, \ell \geq 2$, and every $M>0$, there are $\epsilon_{0}>0$ and $m_{0}>0$ independent of $t$ and $m$ such that for every $m \geq m_{0}$, we have

$$
\left\|e^{-\frac{t}{m} \widetilde{\square}_{b, m}}(\widetilde{x}, \widetilde{y})-\widetilde{\Gamma}_{m}(t, \widetilde{x}, \widetilde{y})\right\|_{C^{l}(\widetilde{X} \times \widetilde{X})} \leq e^{-\frac{m}{t} \epsilon_{0}}, \quad \forall t \in(0, M) .
$$

From Theorem 3.6.4 in [19], we have 
Proposition 3.10. For any $t_{0}>0, \varepsilon>0$ and any $\gamma \in \Gamma, j=1,2, \cdots, N$, there exists $C>0$ such that for any $z \in \widetilde{U}_{\gamma, j}, m \in \mathbb{N}, t>t_{0}$,

$$
\left\|\widetilde{A}_{B_{\gamma, j}, m}(t, z, z)-A_{B_{j}, m}(t, \pi(z), \pi(z))\right\|_{C^{l}\left(\widetilde{U}_{\gamma, j} \times \widetilde{U}_{\gamma, j}\right)} \leq C \exp \left(-\frac{m}{32 t} \varepsilon\right) .
$$

From (3.11) (see (3.31) in [13]), (3.28), (3.29), Proposition 3.10 and the fact that $\widetilde{\psi}_{\gamma, j}=\psi_{j} \circ \pi$, we can easily deduce that

Lemma 3.11. With the above notations and assumptions as in Theorem 3.9, we have

$$
\left\|\widetilde{\Gamma}_{m}(t, \widetilde{x}, \widetilde{x})-\Gamma_{m}(t, \pi(\widetilde{x}), \pi(\widetilde{x}))\right\|_{C^{l}(\widetilde{X} \times \tilde{X})} \leq C \exp \left(-\frac{m}{t} \epsilon_{0}\right) .
$$

From Theorem 3.9, Lemma 3.11 and Theorem 3.5 of [13], we have

Theorem 3.12. For every $\ell \in \mathbb{N}, \ell \geq 2$, and every $M>0$, there are $\epsilon_{0}>0$ and $m_{0}>0$ independent of $t$ and $m$ such that for any $\widetilde{x} \in \widetilde{X}$ and $m \geq m_{0}$, we have

$$
\left\|e^{-\frac{t}{m} \widetilde{\square}_{b, m}}(\widetilde{x}, \widetilde{x})-e^{-\frac{t}{m} \square_{b, m}}(\pi(\widetilde{x}), \pi(\widetilde{x}))\right\|_{C^{l}(\widetilde{X} \times \widetilde{X})} \leq C \exp \left(-\frac{m}{t} \epsilon_{0}\right), \quad \forall t \in(0, M) .
$$

By Theorem 3.2 and Theorem 3.12, we have

Theorem 3.13. With the above notations and assumptions, for every $\epsilon>0$, there are $m_{0}>0$, $\varepsilon_{0}>0$ and $C>0$ such that for all $m \geq m_{0}$, we have

$$
\begin{aligned}
& \left|e^{-\frac{t}{m} \widetilde{\square}_{b, m}}(\widetilde{x}, \widetilde{x})-\sum_{s=1}^{p} e^{\frac{2 \pi(s-1)}{p} m i}(2 \pi)^{-n-1} m^{n} \frac{\operatorname{det}(\dot{\mathcal{R}}) \exp \left(t \gamma_{d}\right)}{\operatorname{det}(1-\exp (-t \dot{\mathcal{R}}))}(\pi(\widetilde{x})) \otimes \operatorname{Id}_{E_{\pi(\widetilde{x})}}\right| \\
& \leq \epsilon m^{n}+C m^{n} t^{-n} e^{\frac{-\varepsilon_{0} m \hat{d}\left(\pi(\widetilde{x}), X_{\text {sing }}\right)^{2}}{t}}, \quad \forall(t, \widetilde{x}) \in \mathbb{R}_{+} \times \widetilde{X}_{\text {reg }} .
\end{aligned}
$$

Recall that since $\Gamma$ acts on $\widetilde{X}$ freely so that $\tilde{X} / \Gamma=X$, hence, we have $\widetilde{X}_{\text {reg }} / \Gamma=X_{\text {reg }}$.

\section{HeAt kernel PROOF}

In this section, we will present the heat kernel proof of the main theorem.

We denote by $\operatorname{Tr}_{\Gamma, q}$ the $\Gamma$-trace of operators acting on $L_{m}^{2}\left(\widetilde{X}, T^{* 0, q} \widetilde{X} \otimes \widetilde{E}\right)$, see Subsection 2.3 or [19, Subsection 3.6.1].

Lemma 4.1. For any $t>0, m \in \mathbb{N}, 0 \leq q \leq n$, we have

$$
\sum_{j=0}^{q}(-1)^{q-j} \operatorname{dim}_{\Gamma} \bar{H}_{b,(2), m}^{j}(\widetilde{X}, \widetilde{E}) \leq \sum_{j=0}^{q}(-1)^{q-j} \operatorname{Tr}_{\Gamma, j}\left[\exp \left(-\frac{t}{m} \widetilde{\square}_{b, m}\right)\right],
$$

with equality for $q=n$.

Proof. Let $E_{\lambda}^{j, m}$ be the spectral resolution of $\widetilde{\square}_{b, m}$ acting on $L_{m}^{2}\left(\widetilde{X}, T^{* 0, q} \widetilde{X} \otimes \widetilde{E}\right)$. We consider the projectors $\left.\left.E^{j, m}(] \lambda_{1}, \lambda_{2}\right]\right)=E_{\lambda_{2}}^{j, m}-E_{\lambda_{1}}^{j, m}$, where $\lambda_{2}>\lambda_{1} \geq 0$. Then, by the Hodge decomposition (3.26) $\left.\left., \sum_{j=0}^{q}(-1)^{q-j} E^{j, m}(] \lambda_{1}, \lambda_{2}\right]\right)$ is the projection on the range of $\left.\left.\bar{\partial}_{b, m} E^{q, m}(] \lambda_{1}, \lambda_{2}\right]\right)$ and thus a positive operator. Hence the $\Gamma$-invariant measure $\sum_{j=0}^{q}(-1)^{q-j} d E_{\lambda}^{j, m}$ is positive on $\{\lambda>0\}$. It follows that

$$
R:=\int_{\lambda>0} e^{-\frac{t}{m} \lambda} \sum_{j=0}^{q}(-1)^{q-j} d E_{\lambda}^{j, m} \geq 0,
$$

and $R$ commutes with $\Gamma$. On the other hand,

$$
\operatorname{Tr}_{\Gamma, j}\left[\exp \left(-\frac{t}{m} \widetilde{\square}_{b, m}\right)\right]=\operatorname{dim}_{\Gamma} \bar{H}_{b,(2), m}^{j}(\widetilde{X}, \widetilde{E})+\operatorname{Tr}_{\Gamma} \int_{\lambda>0} e^{-\frac{t}{m} \lambda} d E_{\lambda}^{j, m} .
$$

By (4.2) and (4.3), we obtain the result. 
Let $\operatorname{Tr}_{q}\left[\exp \left(-\frac{t}{m} \square_{b, m}\right)\right]$ be the trace of the operator $\exp \left(-\frac{t}{m} \square_{b, m}\right)$ acting on $\Omega_{m}^{0, q}(X, E)$. It is well-known that (see Theorem 8.10 in [21])

$$
\operatorname{Tr}_{q}\left[\exp \left(-\frac{t}{m} \square_{b, m}\right)\right]=\int_{X} \operatorname{Tr}_{q}\left[\exp \left(-\frac{t}{m} \square_{b, m}\right)(x, x)\right] d v_{X}(x)
$$

By [19, (3.6.7)] and [19, (3.6.8)], as in (4.4),

Proposition 4.2. We have

$$
\operatorname{Tr}_{\Gamma, q}\left[\exp \left(-\frac{t}{m} \widetilde{\square}_{b, m}\right)\right]=\int_{U} \operatorname{Tr}_{q}\left[e^{-\frac{t}{m} \widetilde{\square}_{b, m}}(\widetilde{x}, \widetilde{x})\right] d v_{\widetilde{X}}(\widetilde{x})
$$

Now we are in a position to give the heat kernel proof of the Morse inequalities for the Fourier components of reduced $L^{2}$ Kohn-Rossi cohomology.

Proof of Theorem 1.1. Denote by $\operatorname{Tr}_{\Lambda^{0, q}}$ the trace on $T^{* 0, q} X$. The basis for $T^{* 0, q} X$ is

$$
\left\{\bar{\omega}^{j_{1}} \wedge \cdots \wedge \bar{\omega}^{j_{q}}: j_{1}<\cdots<j_{q}\right\}
$$

We write for the index $(1, \ldots, q)$

$$
\begin{aligned}
& \exp \left(t \gamma_{d}\right)\left(\bar{\omega}^{1} \wedge \cdots \wedge \bar{\omega}^{q}\right) \\
& =\prod_{j=1}^{q}\left(1+\left(e^{-t a_{j}}-1\right) \bar{\omega}^{j} \wedge \iota_{\bar{\omega}^{j}}\right)\left(\bar{\omega}^{1} \wedge \cdots \wedge \bar{\omega}^{q}\right) \\
& =\sum_{k_{1}<\cdots<k_{q}} c_{k_{1} \ldots k_{q}}(x) \bar{\omega}^{k_{1}} \wedge \cdots \wedge \bar{\omega}^{k_{q}} .
\end{aligned}
$$

From direct calculations, we see that

$$
c_{1 \ldots q}(x)=\exp \left(-t \sum_{j=1}^{q} a_{j}(x)\right) .
$$

Then we have

$$
\operatorname{Tr}_{\Lambda^{0, q}}\left[\exp \left(t \gamma_{d}\right)\right]=\sum_{j_{1}<\cdots<j_{q}} \exp \left(-t \sum_{i=1}^{q} a_{j_{i}}(x)\right)
$$

Hence

$$
\begin{aligned}
& \lim _{t \rightarrow \infty} \frac{\operatorname{Tr}_{\Lambda^{0, q}}\left[\exp \left(t \gamma_{d}\right)\right]}{\operatorname{det}(1-\exp (-t \dot{\mathcal{R}}))} \\
& =\lim _{t \rightarrow \infty} \frac{\sum_{j_{1}<\cdots<j_{q}} \exp \left(-t \sum_{i=1}^{q} a_{j_{i}}(x)\right)}{\prod_{j=1}^{n}\left(1-\exp \left(-t a_{j}(x)\right)\right)}=(-1)^{q} 1_{X(q)},
\end{aligned}
$$


where the function $X(q)$ is defined by 1 on $X(q)$, 0 otherwise. As usual, for $\widetilde{x} \in \widetilde{X}, \pi(\widetilde{x})=x \in$ $X$. It follows from Theorem 3.13, (4.5) and Lemma 4.1 that

$$
\begin{aligned}
& \frac{1}{m^{n}} \sum_{j=0}^{q}(-1)^{q-j} \operatorname{dim}_{\Gamma} \bar{H}_{b,(2), m}^{j}(\widetilde{X}, \widetilde{E}) \\
& \leq \frac{1}{m^{n}} \sum_{j=0}^{q}(-1)^{q-j} \operatorname{Tr}_{\Gamma, q}\left[\exp \left(-\frac{t}{m} \widetilde{\square}_{b, m}\right)\right] \\
& =\frac{1}{m^{n}} \sum_{j=0}^{q}(-1)^{q-j} \int_{U} \operatorname{Tr}_{\Gamma, q}\left[\exp \left(-\frac{t}{m} \widetilde{\square}_{b, m}(\widetilde{x}, \widetilde{x})\right)\right] d v_{\tilde{X}}(\widetilde{x}) \\
& \leq(2 \pi)^{-n-1} \sum_{s=1}^{p} e^{\frac{2 \pi(s-1)}{p} m i} \sum_{j=0}^{q}(-1)^{q-j} \int_{X} \frac{\operatorname{det}(\dot{\mathcal{R}}) \operatorname{Tr}_{\Lambda^{0, q}}\left[\exp \left(t \gamma_{d}\right) \otimes \operatorname{Id}_{E_{x}}\right]}{\operatorname{det}(1-\exp (-t \dot{\mathcal{R}}))} d v_{X}(x) \\
& +\epsilon \sum_{j=0}^{q}(-1)^{q-j} \operatorname{Vol}(X)+C \sum_{j=0}^{q}(-1)^{q-j} \int_{X} t^{-n} e^{\frac{-\varepsilon_{0} m \hat{d}\left(x_{0}, X_{\text {sing }}\right)^{2}}{t}} d v_{X}(x) .
\end{aligned}
$$

Note that $\epsilon$ is arbitrarily small. By the dominant convergence theorem with $t \rightarrow \infty$, we have

$$
\begin{aligned}
& \limsup _{m \rightarrow \infty, p \mid m} \frac{1}{m^{n}} \sum_{j=0}^{q}(-1)^{q-j} \operatorname{dim}_{\Gamma} \bar{H}_{b,(2), m}^{j}(\widetilde{X}, \widetilde{E}) \leq \frac{p r}{(2 \pi)^{n+1}} \sum_{j=0}^{q}(-1)^{q-j} \int_{X(j)}|\operatorname{det}(\dot{\mathcal{R}})| d v_{X}(x), \\
& \limsup _{m \rightarrow \infty} \frac{1}{m^{n}} \sum_{j=0}^{q}(-1)^{q-j} \operatorname{dim}_{\Gamma} \bar{H}_{b,(2), m}^{j}(\widetilde{X}, \widetilde{E})=0, \text { for } \quad p \nmid m .
\end{aligned}
$$

From Definition 2.2, (3.6) and (4.12), we finally get

$$
\begin{aligned}
& \sum_{j=0}^{q}(-1)^{q-j} \operatorname{dim}_{\Gamma} \bar{H}_{b,(2), m}^{j}(\widetilde{X}, \widetilde{E}) \leq \frac{p r m^{n}}{2 \pi^{n+1}} \sum_{j=0}^{q}(-1)^{q-j} \int_{X(j)}\left|\operatorname{det}\left(\mathcal{L}_{x}\right)\right| d v_{X}(x)+o\left(m^{n}\right), \text { for } p \mid m, \\
& \sum_{j=0}^{q}(-1)^{q-j} \operatorname{dim}_{\Gamma} \bar{H}_{b,(2), m}^{j}(\widetilde{X}, \widetilde{E})=o\left(m^{n}\right), \text { for } p \nmid m .
\end{aligned}
$$

Let $q=n$ in (4.1), by applying Theorem 3.13, we obtain for $p \mid m$,

$$
\begin{aligned}
& \frac{1}{m^{n}} \sum_{j=0}^{n}(-1)^{n-j} \operatorname{dim}_{\Gamma} \bar{H}_{b,(2), m}^{j}(\widetilde{X}, \widetilde{E}) \\
& \geq \frac{1}{m^{n}} \sum_{j=0}^{n}(-1)^{n-j} \int_{U} \operatorname{Tr}_{\Gamma, j}\left[\exp \left(-\frac{t}{m} \widetilde{\square}_{b, m}(\widetilde{x}, \widetilde{x})\right)\right] d v_{\widetilde{X}}(\widetilde{x}) \\
& \geq(2 \pi)^{-n-1} p \sum_{j=0}^{n}(-1)^{n-j} \int_{X} \frac{\operatorname{det}(\dot{\mathcal{R}}) \operatorname{Tr}_{\Lambda^{0, j}}\left[\exp \left(t \gamma_{d}\right) \otimes \operatorname{Id}_{E_{x}}\right]}{\operatorname{det}(1-\exp (-t \dot{\mathcal{R}}))} d v_{X}(x) \\
& -\epsilon n \operatorname{Vol}(X)-C n \int_{X} t^{-n} e^{\frac{-\varepsilon_{0} m \hat{d}\left(x_{0}, X_{\text {sing }}\right)^{2}}{t}} d v_{X}(x) .
\end{aligned}
$$

Note that $\epsilon$ is arbitrarily small. By the dominant convergence theorem with $t \rightarrow \infty$, we have (4.15)

$$
\liminf _{m \rightarrow \infty, p \mid m} \frac{1}{m^{n}} \sum_{j=0}^{n}(-1)^{n-j} \operatorname{dim}_{\Gamma} \bar{H}_{b,(2), m}^{j}(\widetilde{X}, \widetilde{E}) \geq \frac{p r}{(2 \pi)^{n+1}} \sum_{j=0}^{n}(-1)^{n-j} \int_{X(j)}|\operatorname{det}(\dot{\mathcal{R}})| d v_{X}(x) .
$$


Then

$$
\liminf _{m \rightarrow \infty, p \mid m} \frac{1}{m^{n}} \sum_{j=0}^{n}(-1)^{n-j} \operatorname{dim}_{\Gamma} \bar{H}_{b,(2), m}^{j}(\widetilde{X}, \widetilde{E})=\frac{p r}{(2 \pi)^{n+1}} \sum_{j=0}^{n}(-1)^{n-j} \int_{X(j)}|\operatorname{det}(\dot{\mathcal{R}})| d v_{X}(x) .
$$

We finally get

$(4.17)$

$$
\sum_{j=0}^{n}(-1)^{n-j} \operatorname{dim}_{\Gamma} \bar{H}_{b,(2), m}^{j}(\widetilde{X}, \widetilde{E})=\frac{p r m^{n}}{2 \pi^{n+1}} \sum_{j=0}^{n}(-1)^{n-j} \int_{X(j)}\left|\operatorname{det}\left(\mathcal{L}_{x}\right)\right| d v_{X}(x)+o\left(m^{n}\right) \text { for } p \mid m .
$$

Then the proof is completed.

\section{REFERENCES}

[1] M. F. Atiyah, Elliptic operators, discrete groups and von Neumann algebras, Astérisque, 32-33 (1976), pp. 43-72

[2] M.-S. Baouendi and L.-P. Rothschild and F.-Treves, CR structures with group action and extendability of CR functions, Invent. Math., 83 (1985), 359-396.

[3] J.-M. Bismut, Demailly's asymptotic Morse inequalities: a heat equation proof, J. Funct. Anal., 72 (1987), 263-278.

[4] L. Boutet de Monvel, Intégration des équations de Cauchy-Riemann induites formelles, Séminaire Goulaouic-Lions-Schwartz 1974-1975; Équations aux derivées partielles linéaires et non linéaires, Centre Math., École Polytech., Paris, 1975, Exp. no. 9, pp. 13.

[5] J.-H. Cheng, C.-Y. Hsiao and I.-H. Tsai, Heat kernel asymptotics, local index theorem and trace integrals for Cauchy-Riemann manifolds with $S^{1}$ action, Mém. Soc. Math. Fr. (N.S.), no.162 (2019), vi+139 pp.

[6] J.-J. Duistermaat and G.-J. Heckman, On the Variation in the Cohomology of the Symplectic Form of the Reduced Phase Space, Invent. Math., 69 (1982), 259-268.

[7] J. P. Demailly, Champs magnétiques et inégalités de Morse pour la d" -cohomologie, Ann. Inst. Fourier, 35 (1985), 189-229.

[8] C. L. Epstein, CR-structures on three dimensional circle bundles, Invent. Math., 109 (1992), 351-403.

[9] E. Getzler, An analoque of Demaillys inequality for strictly pseudoconvex CR manifolds, J. Diff. Geom., 29 (1989), no. 2, 231-244.

[10] A. Grigoryan and L. Saloff-Coste, Dirichlet heat kernel in the exterior of a compact set, Comm. Pure Appl. Math., 55(2002), 93-133.

[11] M. Gromov, M. G. Henkin, M. Shubin, L2 holomorphic functions on pseudo-convex coverings, GAFA, 8 (1998), 552-585.

[12] C.-Y. Hsiao, Szegö kernel asymptotics for high power of CR line bundles and Kodaira embedding theorems on CR manifolds, Memoirs of the American Mathematical Society., 254 (2018), no. 1217, v+142 pp.

[13] C.-Y. Hsiao and R.-T. Huang, The asymptotics of the analytic torsion on CR manifolds with $S^{1}$ action, Comm. Contem. Math., 21 (2019), no.4, 1750094, 35pp .

[14] C.-Y. Hsiao, R.-T. Huang, X. Li and G. Shao, $S^{1}$-equivariant index theorems and Morse inequalities on complex manifolds with boundary. arXiv:1711.05537.

[15] C.-Y. Hsiao and X. Li, Szegö kernel asymptotics and Morse inequalities on CR manifolds with $S^{1}$ action, Asian J. Math., 22 (2018), no.3, 413-450.

[16] C.-Y. Hsiao and X. Li, Morse inequalities for Fourier components of Kohn-Rossi cohomology of CR manifolds with $S^{1}$-action, Math. Z., 284 (2016), no. 1-2, 441-468.

[17] C.-Y. Hsiao and G. Marinescu, Szegö kernel asymptotics and Morse inequalities on CR manifolds, Math. Z., 271 (2012), 509553.

[18] C.-Y. Hsiao and G. Shao, Equidistribution theorems on strongly pseudoconvex domains, Trans. Amer. Math. Soc., 372 (2019) no. 2, 1113-1137.

[19] X. Ma and G. Marinescu, Holomorphic Morse inequalities and Bergman kernels, Progress in Mathematics, 254, Birkhäuser Verlag, Basel, (2007).

[20] G. Marinescu, R. Todor and I. Chiose, $L^{2}$ holomorphic sections of bundles over weakly pseudoconvex coverings, Proceedings of the Euroconference on Partial Differential Equations and their Applications to Geometry and Physics (Castelvecchio Pascoli, 2000), Geom. Dedicata 91 (2002), 23-43. 
[21] J. Roe, Elliptic operators, topology and asymptotic methods, Second edition, Pitman Research Notes in Mathematics Series, 395, Longman, Harlow, (1998).

[22] Y. T. Siu, A vanishing theorem for semipositive line bundles over non-Kähler manifolds, J. Diff. Geom., 19 (1984), 431-452.

[23] M. Shubin, L Riemann=Roch theorem for elliptic operators, GAFA 5(2) (1995), 482-527.

[24] R. Todor, I. Chiose and G. Marinescu, Morse inequalities for covering manifolds, Nagoya Math. J. 163 (2001), 145-165.

Department of Mathematics, National Central University, Chung-Li 320, Taiwan

E-mail address: rthuang@math.ncu.edu.tw

School of Mathematics (Zhuhai), Sun Yat-Sen University, Zhuhai 519082, Guangdong, China E-mail address: shaogk@mail.sysu.edu.cn 